JAN $3 \cap 19 \dddot{30 ̈}$

OSTI

\title{
External Communication FY 1995 Site Support Program Plan WBS 6.10.6
}

W. P. Whiting

Date Published

September 1994

Prepared for the U.S. Department of Energy
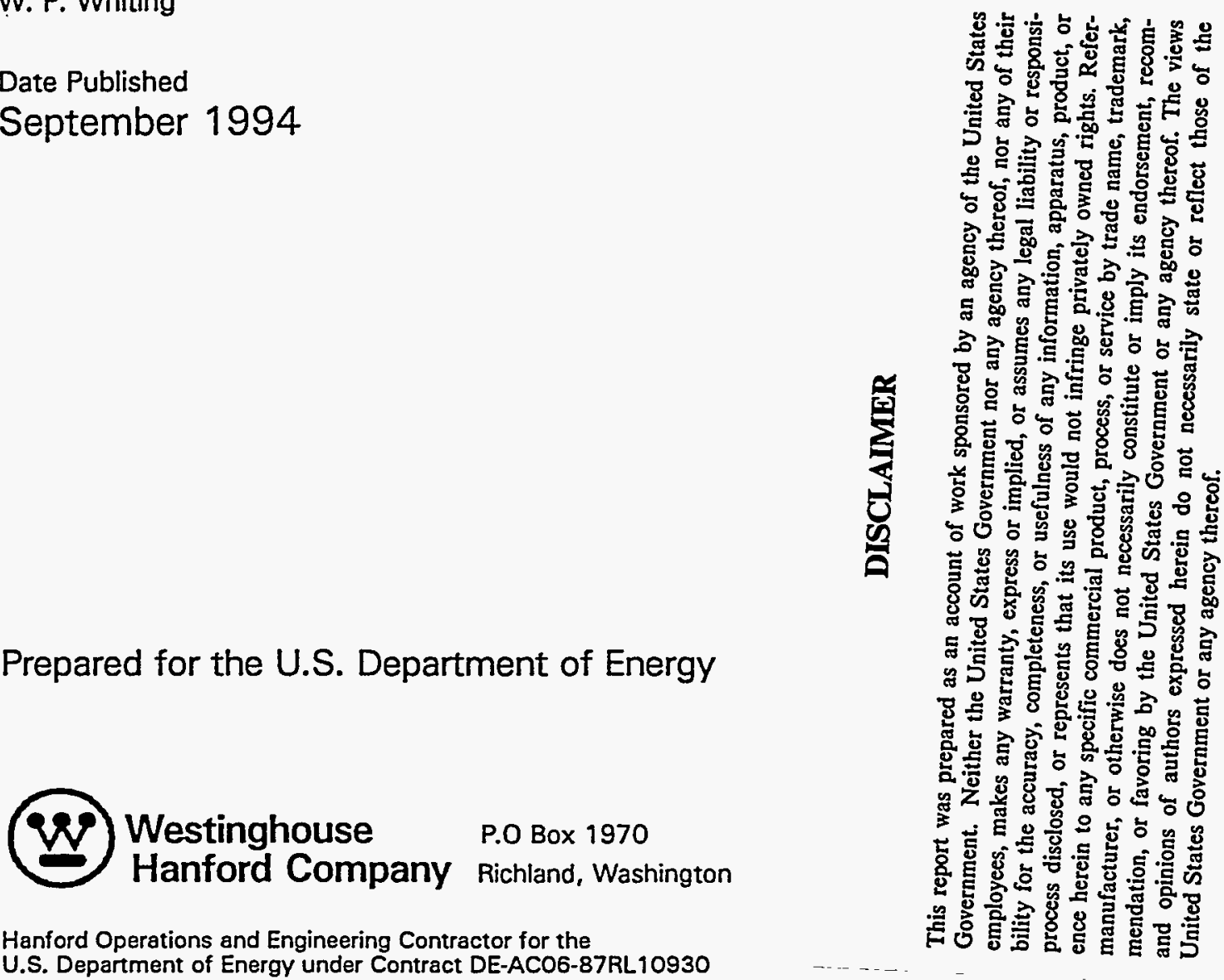


\section{RELEASE AUTHORIZATION}

Document Number: WHC-SP-1132, Rev. 0

Document Title:

External Communication FY 1995 Site Support Program Plan

Release Date:

Pub7 ished September 1994

\section{This document was reviewed following the procedures described in WHC-CM-3-4 and is:}

\section{APPROVED FOR PUBLIC RELEASE}

WHC Information Release Administration Specialist:

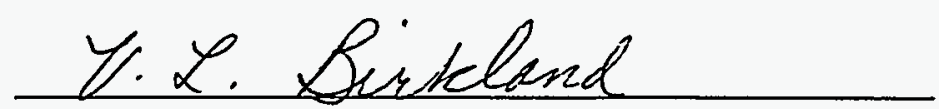

TRADEMARK DISCLAIMER. Reference herein to any specific comercial product, process, or service by trade name, trademark, manufacturer, or otherwise, does not necessarily constitute or imply its endorsement, recommendation, or favoring by the United States Government or any agency thereof or its contractors or subcontractors.

This report has been reproduced from the best available copy. Available in paper copy and microfiche. Printed in the United States of America. Available to the U.S. Department of Energy and its contractors from:

U.S. Department of Energy

Office of Scientific and Technical Information (OSTI)

P.0. Box 62

Oak Ridge, TN 37831

Telephone: (615) 576-8401

Available to the public from:

U.S. Department of Commerce National Technical Information Service (NTIS) 5285 Port Royal Road

Springfield, VA 22161

Telephone: (703) 487-4650 


\title{
Site Support Program Plan Approval Sheet.
}

\author{
6.10.6 External Communications
}

Assistant Manager-Contracting Officer's Representative
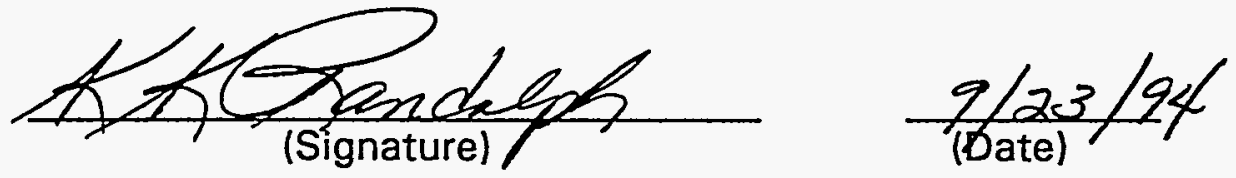

K. K. Randolph, Director RL-Communications

\section{WHC Director \& Program Manager}

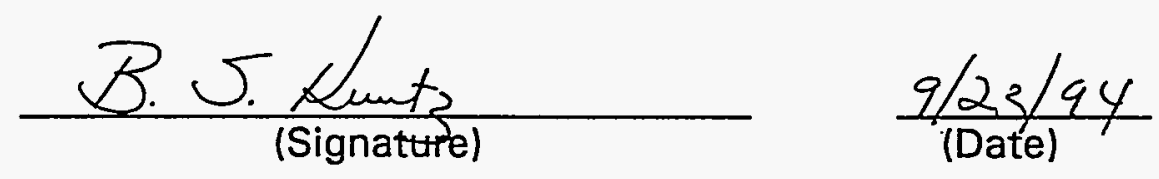

B. S. Kuntz, Director External Communications 


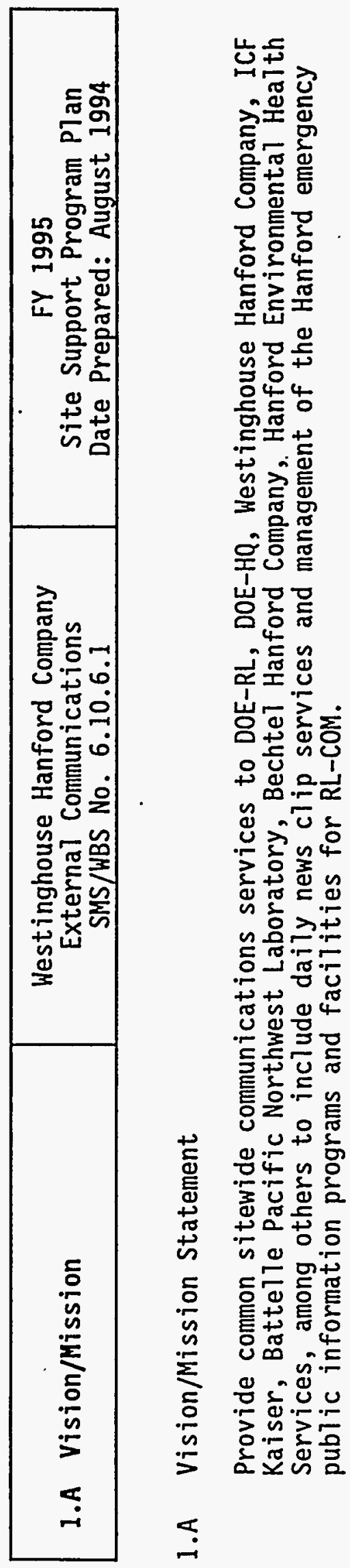




\begin{tabular}{|l|c|c|}
\hline I.A Vision/Mission & $\begin{array}{c}\text { Westinghouse Hanford Company } \\
\text { External Communications } \\
\text { SMS/WBS No.6.10.6.2 }\end{array}$ & $\begin{array}{c}\text { FY 1995 } \\
\text { Site Support Program P1an } \\
\text { Date Prepared: August 1994 }\end{array}$ \\
\hline
\end{tabular}

\section{A Vision/Mission Statement}

Establish a positive working relationship that builds confidence and enhances trust between Hanford and its stakehoiders.

Assist Hanford cleanup progress by facilitating an informed consent process that is sensitive to the values of affected publics, stakeholders, and regulators and assures high-quality public input to decision-making.

Emphasize a proactive communications strategy that is productive and responsive to RL requirements and public/stakeholder values and which insures effective public interface and Hanford progress.

Integrate external communications programs and services into the following WHC projects: 1) Facility Transition, 2) Tank Waste Remediation Systems, 3) Nuclear Spent Fue1, 4) Waste, Analytical \& Environmental Services, and 5) Administration. 


\section{B.1 Internal Assessment Summary}

Westinghouse Hanford Company External Communications SMS/WBS No. 6.10.6.2
FY 1995

Site Support Program Plan

Date Prepared: August 1994

\section{PRIMARY CUSTOMERS}

The primary customers of External Communications are:

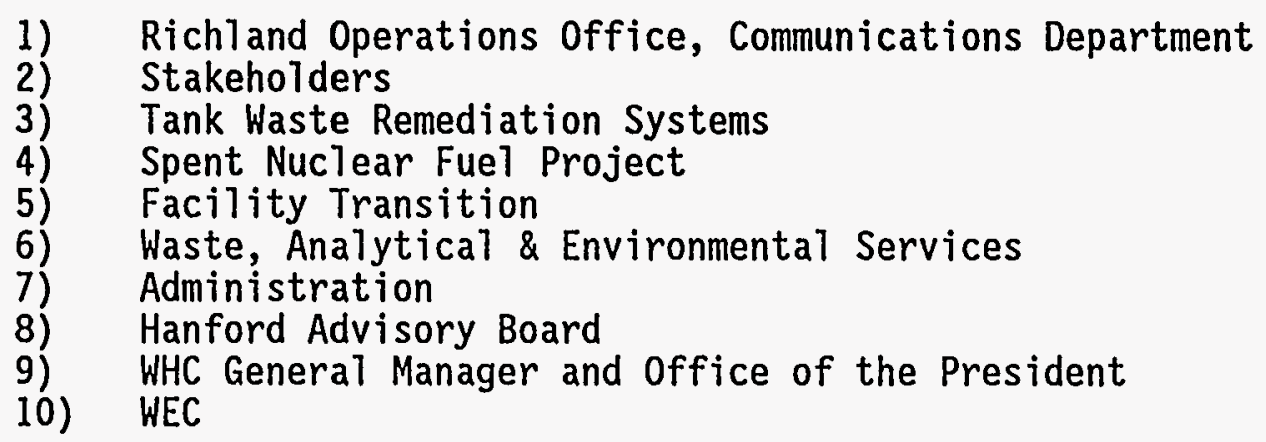

\section{PRODUCTS/SERVICES PROVIDED}

The primary services provided by External Communications are strategic program/project communications planning; comprehensive public involvement; local, regional and national media relations; public information programs and products; community relations; site tours; speakers bureau; crisis communication response; advertising/exhibits; governmental relations/liaison; program communications services; and public communications publications and videos.

\section{MAJOR ACTIVITIES PERFORMED TO DELIVERY PRODUCTS AND SERVICES}

The significant activities performed by External Communications are in four areas: 1) Management; 2) Project Communications Services; 3) Community and Public Outreach; 4) and Public Involvement Services. 


\section{COST OF PERFORMING SERVICES}

The cost of performing external communications services for fiscal year 1995 is $\$ 3.9$ million from indirect funds. Four customers (Tank Waste Remediation Systems, Spent Nuclear Fuel Project, Facility Transition and Waste, Analytical and Environment Services are expected to direct fund information products specific to their projects.)

\section{UNIT COST OF SERVICES}

The unit cost of products and services for External Communications in FY94 is not applicable.

\section{EVALUATION OF MAJOR ACTIVITIES (COST, VALUE-ADDED, ETC.)}

External Communications activities provide value to the company, its projects, and DOE by achieving compliance to more than 30 DOE orders, DOE directives, policies, state and federal laws. Through the systematic development of informed consent involving Hanford management, stakeholders, the general public and regulators, Hanford cleanup is better able to proceed in concert with the values of the involved parties.

External Communications provides further efficiencies as the single point of contact for media interactions which otherwise would be scattered throughout WHC programs.

Enhanced efficiency is expected from the realignment of multi-purpose communication teams which are dedicated to five key programmatic areas: TWRS Communications, Transition Facilities Communications, Spent Fuels Communications, Waste, Analytical and Environmental Services Communications, and Program Communications Services.

\section{FACTORS WHICH INFLUENCE RESOURCE CONSUMPTION (CHANGES TO COST AND VOLUME)}

The factors that influence cost and volume for External Communications are significant. While External Communications can plan for an expected level of activity that is based on historical levels, it is impossible to predict nor control the events which will require direct communications response. 
For example, the recent identification by the Secretary of Energy of the three tribes as co-regulators of Hanford directly impacts the level of effort and cost of achieving planned public involvement.

\section{FACTORS THAT INFLUENCE CHANGES IN TYPES OR NATURE OF PRODUCTS and SERVICES}

The factors that influence changes in the nature of External Communications services are directly related to management decisions both within WHC and at DOE and stakeholder expectations.

For example, stakeholder interest in an issue will require increased information and interaction be provided. Technological influences are also present as demonstrated by the unanticipated FY94 request to implement a multimedia information program. Integration into the information highway is expected to be an influence in FY95.

\section{ASSESSMENT OF PROBLEMS, ISSUE AND OPPORTUNITIES}

External Communications faces a significant challenge to streamline its operation, be more cost effective and provide services directly to key WHC programs. At risk, however, are the efficiencies realized as a centralized overhead organization. To ensure both efficiency and effectiveness are maintained, the department will retain both an overhead function and a program services cost center. Services provided directiy to projects will be paid for by the projects.

Maintaining communications between stakeholders and WHC program management is at a criticai stage at this time. Without the confidence of stakeholders, the pubi ic and regulators that cleanup progress is being made safely and cost effectively, future resources may be restricted and third party intervention likety.

The leadership of both the Department of Energy and Westinghouse have committed to assure public input to the decision-making process. The systematic development of informed consent process is now assuring open, two-way communications with Hanford stakeholders, among others. Without the facilitation of this process by External Communications, customer expectations will not be met.

Crucial to progress and informed consent is information. New and innovative communications strategies and tools responding to the information needs of a technologically driven audience are being developed. For example, the CD ROM communications products have proven highly successful in their demonstrations, but further development is needed. Internet capability is anticipated to be a required information service. 


\section{B.2 External Assessment Summary}

Westinghouse Hanford Company External Communications SMS/WBS No. 6.10.6.2
FY 1995

Site Support Program Plan Date Prepared: August 1994

\section{SUMMARY OF CUSTOMER REQUIREMENTS (NEEDS)}

The issues facing Westinghouse Hanford External Communications can be summarized into three broad categories: 1) Assure public involvement in Hanford decision making; 2) Make public access to information easier; 3) Consult with Native American tribes as sovereign nations to ensure Hanford related tribal goals are incorporated in Hanford decision making.

\section{Hanford Site Communications Plan}

A formal process to establish common goals and objectives with the DOE customer, in partnership with the other contractor public affairs directors, was started in February 1994. To support this process, a formal meeting was held jointly with the Richland Operations Manager and each contractor principal to discuss expectations concerning communications. Additionally, the DOE-HQ Strategic Plan and the Hanford Summit II commitments were reviewed to define communications requirements and impacts. The draft FY 1994-95 Hanford Site Communications Plan reflects these inputs. It also focuses on the strategies and actions that the RL and contractor communication staffs are using to increase stakeholder and Native American understanding of and involvement in DOE's Hanford decisions. 
The External Communications Department has recentiy restructured to align directly with the four major Hanford cleanup programs. However, the fundamental goals, objectives, and missions remain unchanged. The management goal for the Department is to assure public involvement in Hanford decision making, make public access to information easier and consult with Native Americans on Hanford decisions.

The organizational goals of the Department are to be recognized as a team of professionals who are proactive, responsive to customer needs, and are experts in their field.

The objectives of the Department are to develop and implement program-driven, external communications services that:

- Increase public understanding of the Hanford environmental and waste management mission;

- Increase public involvement in the decision-making process through open, two-way communications with all stakeholders and WHC/DOE program management;

- More frequently communicate program achievements and issues;

- Establish and maintain effective working relationships with local, regional, and national media to ensure accurate and timely communication of key Hanford issues.

- Respond promptly to information requests from all stakeholders, media, regulators, government and elected officials, tribes, labor leaders, community leaders, and business Teaders;

- Establish community goodwill and recognition that Hanford is a good neighbor; 


\section{D Strategies}

Westinghouse Hanford Company

External Communications SMS/WBS No. 6.10.6.2
FY 1995

Site Support Program Plan Date Prepared: August 1994

\section{CUSTOMER SUPPORT STRATEGIES}

Multi-purpose teams capable of providing a full range of communication services will be refined and dedicated to four key cleanup programs and one administrative area. Services provided will include strategic planning, public involvement, public information and media information.

Services essential for the functioning of the Hanford Advisory Board and Tri-Party Agreement public involvement requirements will be dedicated to those entities.

Customer needs and satisfaction will be assessed through one-on-one interactions with DOE-RL and stakeholders and through group meetings as needed.

\section{PRODUCTION STRATEGIES}

Information required is determined by the cognizant communication group whether it be the manager or the project team. Development of the information is then "contracted" with the appropriate source. For efficiency, External Communications has developed an internal Information Products Center of

Excellence which serves as a full service resource for electronic and printed information products.

\section{ORGANIZATION and MANAGEMENT STRATEGIES}

The organization and management strategies are carried out though the teams described in the "Customer Support Strategies" section. The team will be supported by the department as a whole which will provide broad services i.e. Speakers Bureau, tours, information product development, government relations, etc. Managers will serve as "coaches" to the teams as well as senior experts in their areas of expertise. Department activities will be coordinated both in the project team level and at a management leve1. Teams will have the flexibility to "borrow" resources temporarily from other teams to address high volume workload issues. 


\begin{tabular}{|l|c|c|}
\hline 1.E Assumptions & $\begin{array}{c}\text { Westinghouse Hanford Company } \\
\text { External Communications } \\
\text { SMS/WBS No.6.10.6.2 }\end{array}$ & $\begin{array}{c}\text { FY } 1995 \\
\text { Site Support Program P1an } \\
\text { Date Prepared: August 1994 }\end{array}$ \\
\hline
\end{tabular}

The External Communications Department's goals, objectives and strategies are driven primarily by the Hanford Strategic Plan and the Tri-Party Agreement, both of which call for Hanford to build partnerships with its stakeholders, and the commitment by Department of Energy and Westinghouse leaders to assure stakeholder participation in Hanford's decision-making process.

The draft Hanford Site Communications Plan also provides a basis with its emphasis on public 'participation and feedback, enhancing public understanding of Hanford's mission and making public access to information and facilities easier.

The development of the department's goals, objectives and strategies also assumes that staffing will be consistent with funding levels; funding will be received from the ERC for communications services provided to it; and the process through which the three co-regulator tribes will be involved in decision-making will be clarified. 


\section{F Issues and Constraints}

Westinghouse Hanford Company

External Communications SMS/WBS No. 6.10.6.2

Site Support Program Plan

Date Prepared: August 1994

The External Communications Department's achievement of its mission will depend greatly on something beyond its control: the ability of the Department of Energy and Westinghouse to prevent unanticipated events which damage working relationships and reduce stakeholder confidence in Hanford.

Management decisions intended to be positive also may become an issue at the External Communications level. A key example is the recent decision about tribal interaction and involvement in the decisionmaking process, which may require more public involvement and other communications support than has been anticipated.

Providing direct communications response to an unanticipated event will likely use resources earmarked for other planned products and services. There is little choice, however, for an ineffective communications response likely would add to the damage caused by the event or decision to which the department was responding. 


\section{G Performance Measures}

Hestinghouse Hanford Company External Communications SMS/WBS No. 6.10.6.2
FY 1995

Site Support Program Plan Date Prepared: August 1994

The performance of External Communications Department strategies will be measured through informal surveys of stakeholder perceptions and the reflection of those perceptions in the department's planning; the strength of stakeholder partnerships and degree to which those partnerships contribute to visibility, understanding and achievement of Hanford's mission' and the adoption of new practices learned from these interactions.

Performance measures also include the number of new communication products; number of ineffective products eliminated; amount of proactive public information provided; time needed to respond to inquiries; and number of customers served. 


\begin{tabular}{|l|c|c|}
\hline 2. A.1 & Westinghouse Hanford Company & $\begin{array}{c}\text { FY } 1995 \\
\text { Work Breakdown Structure and } \\
\text { Responsibility Assignment Matrix }\end{array}$ \\
\hline
\end{tabular}

RL WBS Activity Title Manager * Org. \& Number

6.10 .6

6.10.6.1

WBS700

WBS500

6.10 .6 .2

\begin{tabular}{|c|c|}
\hline WBS 100.1 & $\begin{array}{l}\text { Public Involvement, } \\
\text { Coordination }\end{array}$ \\
\hline WBS100.2 & $\begin{array}{l}\text { Public Involvement, } \\
\text { Strategic Analysis }\end{array}$ \\
\hline WBS100.3 & Public Involvement, Liaison \\
\hline WBS300 & Media Relations \\
\hline WBS400.1 & Community Relations \\
\hline WBS400.2 & Tribal Relations \\
\hline WBS500 & Information Products Center \\
\hline WBS900 & Site Tours \\
\hline WBS1100 & Public Information \\
\hline WBS1200 & Strategic Planning \\
\hline
\end{tabular}

\section{External Communications}

- Site Communications Services Nows Clips

Joint Information Center

Multi Media

Technical Library Assessment

Hanford News Clips

Emergency Public Information

Information Products Center

\section{TWRS Communications}

\section{B.S. Kuntz}

B.S. Kuntz

External Communications

External Communications (01100)

P.M. Phelps

B.S. Kuntz

P.M. Phelps

B.S. Kuntz

P.S. Hale

P.S. Hale

P.S. Hale

P.M. Phelps

D.J. Dillman

D.J. Dillman

P.M. Phelps

D.J. Dillman

P.S. Hale/P.M. Phelps

B.S. Kuntz
Project Communications Services (01110)

External Communications (01100)

Project Communications Services (01110)

\section{External Communications}

Public Involvement Services (01130)

Public Involvement Services (01130)

Public Involvement Services (01130)

Project Communication Services (01110)

Community \& Public Outreach (01120)

Community \& Public Outreach (01120)

Project Communications Services (01110)

Community \& Public Outreach (01120)

Public Involvement/Project

$$
\text { Communications (01130/01110) }
$$

External Communications (01100) 
WBS100.1

WBS 100.2

WBS 100.3

WBS300

WBS400.1

WBS400.2

WBS500

WBS900

WBS 1100

WBS 1200

6.10.6.2

(01100)

WBS 100.1

WBS100.2

WBS100.3

WBS300

WBS400.1

WBS400.2

WBS500

WBS900

WBS 1100

WBS 1200
Environmental Communications

P.S. Hale

$$
\text { Coordination }
$$

Public Involvement, Strategic Analysis

Public Involvement, Liaison

Media Relations

Community Relations

Tribal Relations

Information Products Center

Site Tours

Public Information

Strategic Planning

Facility Transition Communications

Public Involvement,

$$
\text { Coordination }
$$

Public Involvement,

Strategic Analysis

Public Involvement, Liaison

Media Relations

Community Relations

Tribal Relations

Information Products Center

Site Tours

Public Information

Strategic Planning

Spent Nuclear Fuel

Project Communications
P.S. Hale

P.S. Hale

P.M. Phelps

D.J. Dillman

D.J. Dillman

P.M. Phelps

D.J. Dillman

P.S. Hale/P.M. Phelps

B.S. Kuntz

B.S. Kuntz

P.S. Hale

P.S. Hale

P.S. Hale

P.M. Phelps

D.J. Dillman

D.J. Dillman

P.M. Phelps

D.J. Dillman

P.S. Hale/P.M. Phelps

B.S. Kuntz

Communications $(01130 / 01110)$
External Communicati

B.S. Kuntz
External Communications (01100)

Public Involvement Services (01130)

Püblic Involvement Services 1011301

Public Involvement Services (01130)

Project Communication Services (01110)

Community \& Public Outreach 101120 )

Project Communications Services (01110)

Community \& Public Outreach (01120)

Communications (01130/01110)

External Communications (01100)

\section{External Communications}

Public Involvement Services (01130)

Public Involvement Services (01130)

Public Involvement Services (01130)

Project Communication Services (01110)

Community \& Public Outreach (01120)

Community \& Public Outreach (01120)

Project Communications Services (01110)

Community \& Public Outreach (01120)

External Communications 1011001

External Communications 

WBS100.1 Public Involvement,
Coordination

WBS100.2

WBS100.3

WBS300

WBS400.1

WBS400.2

WBS500

WBS900

WBS1100

WBS1200

6.10.6.2

WBS300

WBS500

WBS900

WBS1200

6.10.6.2

WBS100.1

WBS100.2

WBS100.3

WBS300

WBS400.1

WBS400.2

WBS500
Public Involvement,

- Strategic Analysis

Public Involvement, Liaison

Media Relations

Community Relations

Tribal Relations

Information Products Center

Site Tours

Public Information

Strategic Planning

Project Communications Services

Media Relations

Information Products Center

Site Tours

Strategic Planning

Public Involvement Services Hanford Advisory Board Tri-Party Agreoment

\section{Public involvement,}

$$
\text { Coordination }
$$

Public Involvement,

$$
\text { Strategic Analysis }
$$

Public Involvement, Liaison

Media Relations

Community Relations

Tribal Relations

Information Products Center
P.S. Hale

Public Involvement Services (01130)
P.S. Hale

P.S. Hale

P.M. Phelps

D.J. Dillman

D.J. Dillman

P.M. Phelps

D.J. Dillman

P.S. Hale/P.M. Phelps

Communications (01130/01110)

B.S. Kuntz

B.S. Kuntz

P.M. Phelps

P.M. Phelps

D.J. Dillman

B.S. Kuntz

B.S. Kuntz

P.S. Hale

P.S. Hale

P.S. Hale

P.M. Phelps

D.J. Dillman

D.J. Dillman

P.M. Phelps
Public Involvement Services (01130)

Public Involvement Services (01130)

Project Communication Services (01110)

Community \& Public Outreach (01120)

Community \& Public Outreach (01120)

Project Communications Services (01110)

Community \& Public Outreach (01120)

Public Involvement/Project

External Communications (01100)

External Communications (01100)

Project Communication Services 101110

Project Communications Services (01110)

Community \& Public Outreach (01120)

External Communications (01100)

External Communications (01100)

Public Involvement Services 1011301

Public Involvement Services (01130)

Public Involvement Services 101130

Project Communication Services $(011101$

Community \& Public Outreach(01120)

Community \& Public Outreach (01120)

Project Communications Services (01110) 


$\begin{array}{ll}\text { WBS900 } & \text { Site Tours } \\ \text { WBS1100 } & \text { Public Information }\end{array}$

WBS1200 Strategic Planning

\subsubsection{2}

\begin{tabular}{|c|c|}
\hline WBS100.1 & $\begin{array}{l}\text { Public Involvement, } \\
\text { Coordination }\end{array}$ \\
\hline WBS100.2 & $\begin{array}{l}\text { Public Involvement, } \\
\text { Strategic Analysis }\end{array}$ \\
\hline WBS100.3 & Public Involvement, Liaison \\
\hline WBS300 & Media Relations \\
\hline WBS400.1 & Community Relations \\
\hline WBS400.2 & Tribal Relations \\
\hline WBS500 & Information Products Center \\
\hline WBS900 & Site Tours \\
\hline WBS1100 & Public Information \\
\hline \multirow[t]{2}{*}{ WBS1200 } & Strategic Planning \\
\hline & Executive Communications \\
\hline WBS100.2 & Public Involvement, Strategic Analysis \\
\hline WBS400.1 & Community Relations \\
\hline WBS400.2 & Tribal Relations \\
\hline WBS500 & Information Products Center \\
\hline WBS600 & Government Relations \\
\hline WBS900 & Site Tours \\
\hline WBS1000 & Speakers Bureau \\
\hline WBS 1200 & Strategic Planning \\
\hline
\end{tabular}

D.J. Dillman

P.S. Hale/P.M. Phelps

B.S. Kuntz

B.S. Kuntz

P.S. Hale

P.S. Hale

P.S. Hale

P.M. Phelps

D.J.'Dillman

D.J. Dillman

P.M. Phelps

D.J. Dillman

P.S. Hale/P.M. Phelps

B.S. Kuntz

B.S. Kuntz

P.S. Hale

D.J. Dillman

D.J. Dillman

P.M. Phelps

D.J. Dillman

D.J. Dillman

D.J. Dillman

B.S. Kuntz
Community \& Public Outreach (01120)

Public Involvement/Project

Communications (01130/01110)

External Communications (01100)

\section{External Communications (01100)}

Public Involvement Services (01130)

Public Involvement Services (01130)

Public Involvement Services (01130)

Project Communication Services (01110)

Community \& Public Outreach (01120)

Community \& Public Outreach (01120)

Project Communications Services 101110

Community \& Public Outreach (01120)

Public Involvement/Project

Communications (01130/01110)

External Communications (01100)

\section{External Communications (01100)}

Public Involvement Services (01130) Community \& Public Outreach (01120)

Community \& Public Outreach (01120)

Project Communications Services (01110)

Community \& Public Outreach (01120)

Community \& Public Outreach (01120)

Community \& Public Outreach (01120)

External Communications (01100) 


\begin{tabular}{|c|c|c|}
\hline 2.A.2 Description of Activities & $\begin{array}{l}\text { Westinghouse Hanford Company } \\
\text { RL WBS \# } 6.10 .6 \\
\text { External Communications }\end{array}$ & $\begin{array}{l}\text { FY } 1995 \\
\text { Site Support Program Plan } \\
\text { Date Prepared: August } 1994\end{array}$ \\
\hline ACTIVITY NUMBER \& TITLE & \multicolumn{2}{|c|}{ DESCRIPTION } \\
\hline $\begin{array}{l}\text { WBS100.1 Public Involvement, } \\
\text { Coordination }\end{array}$ & \multicolumn{2}{|c|}{$\begin{array}{l}\text { Plan, coordinate, and conduct public involvement activities which } 1) \text { support program decisions } \\
\text { and 2) assure compliance with the spirit and intent of environmental laws and agreements with } \\
\text { regulators and stakeholders. }\end{array}$} \\
\hline $\begin{array}{l}\text { WBS100.2 Public Involvement, } \\
\text { Strategic Analysis }\end{array}$ & \multicolumn{2}{|c|}{$\begin{array}{l}\text { Facilitate the strategic analysis process that identifies key decisions that need stakeholder } \\
\text { participation. }\end{array}$} \\
\hline $\begin{array}{l}\text { WBS100.3 Public Involvement, } \\
\text { Liaison }\end{array}$ & \multicolumn{2}{|c|}{$\begin{array}{l}\text { Serve as WHC liaison with stakeholders and special interest groups. Arrange appropriate } \\
\text { interaction forums. Initiate the sharing of project status information with stakeholders and } \\
\text { special interest groups }\end{array}$} \\
\hline WBS200 Hanford Advisory Board & \multicolumn{2}{|c|}{$\begin{array}{l}\text { Provide public involvement and administrative support to DOE-RL in support of the Hanford } \\
\text { Advisory Board and its four subcommittees. Activities include preparing draft issue papers, } \\
\text { logistics, meeting notes and records, cost accounting, travel services, and meeting } \\
\text { management. }\end{array}$} \\
\hline WBS300 Media Relations & \multicolumn{2}{|c|}{$\begin{array}{l}\text { Provide comprehensive media relations services to WHC programs. Research, develop, } \\
\text { distribute news releases, fact sheets, and visual products concerning technical } \\
\text { accomplishments, events and issues. Represent WHC as company spokesperson to the media. } \\
\text { Manage press conferences and special media events. }\end{array}$} \\
\hline WBS400.1 Community Relations & \multicolumn{2}{|c|}{$\begin{array}{l}\text { Maintain open, two way communications with community leaders, site neighbors, regional } \\
\text { publics, stakeholders, and elected officials. }\end{array}$} \\
\hline WBS400.2 Tribal Relations & \multicolumn{2}{|c|}{ Maintain open, two-way communications with affected tribes. } \\
\hline WBS500 Information Products Center & \multicolumn{2}{|c|}{$\begin{array}{l}\text { Produce written, electronic and visual information products which clearly communicates cleanup } \\
\text { progress and issues. }\end{array}$} \\
\hline WBS600 Government Relations & \multicolumn{2}{|c|}{$\begin{array}{l}\text { Monitor legislative and congressional activities that relate to Hanford programs. Coordinate } \\
\text { congressional and legislative briefings. Conduct site tours for elected officials and their staff } \\
\text { members. Respond to requests for information. }\end{array}$} \\
\hline
\end{tabular}




\begin{tabular}{|l|l|}
\hline WBS700 Emergency Public Information & $\begin{array}{l}\text { Coordinate the Hanford Site Emergency Public Information Program to include management of } \\
\text { the Hanford Joint Information Center. Assure compliance to DOE Order 5500.4B and other } \\
\text { related guidance. }\end{array}$ \\
\hline WBS800 Hainford News Clips & $\begin{array}{l}\text { Provide daily newspaper clippings to DOE-RL and site contractors concerning Hanford related } \\
\text { newspaper articles. }\end{array}$ \\
\hline WBS900 Site Tours & $\begin{array}{l}\text { Coordinate site tours to include publishing agendas, badging, access requests, program } \\
\text { coordination, briefer selection, transportation, briefing material preparation and reports. }\end{array}$ \\
\hline WBS1000 Speakers Bureau & $\begin{array}{l}\text { Coordinate the Hanford Speakers Bureau Program including developing presentations, } \\
\text { scheduling presentations, training and providing follow up information. }\end{array}$ \\
\hline WBS1100 Public Information & $\begin{array}{l}\text { Provide proactive information to the general public and stakeholders. Respond formally and } \\
\text { informally to public inquiries, Research, develop, distribute fact sheets, letters and visual } \\
\text { products to fulfill requests. }\end{array}$ \\
\hline WBS1200 Strategic Planning & $\begin{array}{l}\text { Provide a proactive communications strategy that is productive and responsive to RL } \\
\text { requirements and public/stakeholder needs and values which insures effective public interface } \\
\text { and progress at Hanford. }\end{array}$ \\
\hline \hline
\end{tabular}




\begin{tabular}{|c|c|c|c|c|}
\hline \multirow{2}{*}{\multicolumn{2}{|c|}{$\frac{2.8 .1 \text { Milostone List - NA }}{\text { Millostone }}$}} & \multirow{3}{*}{$\begin{array}{c}\text { WBS } \\
\text { Number }\end{array}$} & $\begin{array}{l}\text { Westinghouse Hanford Company } \\
\text { External Communications } \\
\text { SMS/WBS No. }\end{array}$ & $\begin{array}{l}\text { FY } 1995 \\
\text { Site Support Program. Plan } \\
\text { Date Prepared: August } 1994\end{array}$ \\
\hline & & & \multirow{2}{*}{\multicolumn{2}{|c|}{ Milestone Description }} \\
\hline Type & Number & & & \\
\hline \multicolumn{5}{|l|}{ NA } \\
\hline & & & & \\
\hline & & & & \\
\hline & & & & \\
\hline & & & & \\
\hline & & & & \\
\hline & & & & \\
\hline & & & & \\
\hline & & & & \\
\hline & & & & \\
\hline
\end{tabular}




\begin{tabular}{|c|c|c|c|c|c|c|c|}
\hline $\begin{array}{l}\text { 2.C.1.2 } \\
\text { Source }\end{array}$ & Cost Baseline by Funding & \multicolumn{4}{|c|}{$\begin{array}{l}\text { Westinghouse Hanford Company } \\
\text { External Communications } \\
\text { SMS/WBS No. }\end{array}$} & \multicolumn{2}{|c|}{$\begin{array}{c}\text { FY } 1995 \\
\text { SSPP } \\
\text { Date Prepared: } 8 / 94\end{array}$} \\
\hline \multicolumn{8}{|c|}{ FY 1994 COST BASELINE (Dollars in Thousands) } \\
\hline WBS \# & Title & Dept. OH & CSP/Oversight & SLP & $G \& A$ & Direct $\$$ & TOTAL \$S \\
\hline & & $\therefore$ & & & & & \\
\hline & & & & & & & \\
\hline & & \%? & & & & & \\
\hline & & ४४४: & & & & & \\
\hline & & ४৫\%: & & & & & \\
\hline & & क्ष & & & & & \\
\hline & & ४४। & & & & & \\
\hline \multicolumn{8}{|c|}{ TOTALS } \\
\hline \multicolumn{8}{|c|}{ FY 1995 COST BASELINE (Dollars in Thousands) } \\
\hline WBS\# & Title & Dept. OH & CSP/Oversight & SLP & G \& A & Direct $\$$ & Total $\$$ \\
\hline $\begin{array}{l}6.10 .6 . \\
1 \\
\end{array}$ & Site Communications Services & अै: & 631 & & & & 631 \\
\hline $\begin{array}{l}6.10 .6 . \\
2 \\
\end{array}$ & TWRS Communications & \&. & & & 595.4 & 160 & 755.4 \\
\hline$\frac{6.10 .6 .}{2}$ & TrP Communications & भ०षै & & & 184.9 & 82 & 266.9 \\
\hline $\begin{array}{l}6.10 .6 \\
2\end{array}$ & Spent Fuel Communications & मै। & & & 55.1 & 233.9 & 289 \\
\hline
\end{tabular}




\begin{tabular}{|c|c|c|c|c|c|c|c|}
\hline $\begin{array}{l}6.10 .6 \\
2\end{array}$ & $\begin{array}{l}\text { Waste, Analytical and Environmental } \\
\text { Communications }\end{array}$ & मे & & & 196.6 & 106 & 302.6 \\
\hline $\begin{array}{l}6.10 .6 \\
2\end{array}$ & Project Communications Services & औ? & & & 957 & & 957 \\
\hline $\begin{array}{l}6.10 .6 \\
2\end{array}$ & Community/Public Outreach & मे? & & & 660.3 & 61 & 721.3 \\
\hline $\begin{array}{l}6.10 .6 \\
2\end{array}$ & Public Involvement & 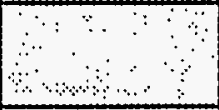 & & & 399 & & 399 \\
\hline $\begin{array}{l}6.10 .6 \\
2\end{array}$ & Executive Communications & $\because$ & & & 966.8 & & 966.8 \\
\hline & TOTALS & $-0-\because$ & 631 & -0 & $4,015.1$ & 642.9 & 5,289 \\
\hline
\end{tabular}

- Direct funding details/are found indirect program long range planning documents and are not included in this SSPP.

\begin{tabular}{|l|c|c|}
\hline & Westinghouse Hanford Company & $\cdot$ \\
$\begin{array}{l}\text { 2.C.1.2 Cost Baseline by Funding } \\
\text { Source }\end{array}$ & External Communications & FY 1995 \\
SSPP & \\
\hline
\end{tabular}

FY 1996 COST BASELINE (Dollars in Thousands)

\begin{tabular}{|c|c|c|c|c|c|c|c|}
\hline WBS \# & Title & Dept OH & CSP/Oversight & SLP & $G \& A$ & Direct $\$$ & TOTAL \$s \\
\hline $\begin{array}{l}6.10 .6 . \\
1\end{array}$ & Site Communications Services & - & 631 & & & & 631 \\
\hline $\begin{array}{l}6.10 .6 . \\
2\end{array}$ & TWRS Communications & 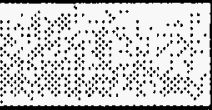 & & & 612.7 & 164.6 & 777.3 \\
\hline $\begin{array}{l}6.10 .6 . \\
2\end{array}$ & TrP Communications & ४ै। & & & 190.3 & 84.4 & 274.7 \\
\hline $\begin{array}{l}6.10 .6 . \\
2\end{array}$ & Spent Fuel Communications & ४ & & & 56.7 & 240.7 & 297.4 \\
\hline
\end{tabular}




\begin{tabular}{|c|c|c|c|c|c|c|}
\hline $\begin{array}{l}6.10 .6 . \\
2 \\
\end{array}$ & $\begin{array}{l}\text { Waste, Analytical and Environmental } \\
\text { Communications }\end{array}$ & ४ & & 202.5 & 109.1 & 311.4 \\
\hline $\begin{array}{l}6.10 .6 \\
2 \\
\end{array}$ & Project Communications Services & अ & & 984.8 & - & 984.8 \\
\hline $\begin{array}{l}6.10 .6 . \\
2\end{array}$ & Community Public Outreach & औ। & & 679.4 & 62.8 & 742.2 \\
\hline $\begin{array}{l}6.10 .6 \\
2 \\
\end{array}$ & Public Involvement & $\therefore \quad: \quad 3 \quad \vdots$ & & 410.6 & & 410.6 \\
\hline $\begin{array}{l}6.10 .6 . \\
2\end{array}$ & Executive Communications & । & & 994.8 & & 994.8 \\
\hline & TOTALS & an & 631 & $4,131.6$ & 661.6 & $5,424.2$ \\
\hline
\end{tabular}

FY 1997 COST BASELINE (Dollars in Thousands)

\begin{tabular}{|c|c|c|c|c|c|c|c|}
\hline WBS\# & Title & Dept:OH & CSP/Oversight & SLP & G \& A & Direct \$ & Total \$ \\
\hline $\begin{array}{l}6.10 .6 \\
1\end{array}$ & Site Communications Services & $\therefore$ & 631 & & & & 631 \\
\hline $\begin{array}{l}6.10 .6 \\
2\end{array}$ & TWRS Communications & +e & & & 631.7 & 169.8 & 801.5 \\
\hline $\begin{array}{l}6.10 .6 \\
2\end{array}$ & TrP Communications & ২, & & & 196.2 & 87 & 283.2 \\
\hline $\begin{array}{l}6.10 .6 . \\
2\end{array}$ & Spent Fuel Communications & 3 & & & 58.5 & 248.2 & 306.7 \\
\hline $\begin{array}{l}6.10 .6 \\
2\end{array}$ & $\begin{array}{l}\text { Waste, Analytical and Environmental } \\
\text { Communications }\end{array}$ & अे & & & 208.6 & 112.5 & 321.1 \\
\hline $\begin{array}{l}6.10 .6 . \\
2\end{array}$ & Project Communications Services & ४ै। ? & & & $1,015.4$ & & $1,015.4$ \\
\hline $\begin{array}{l}6.10 .6 . \\
2\end{array}$ & Community Public Outreach & ঋ। & & & 700.6 & 64.7 & 765.3 \\
\hline
\end{tabular}




$$
\text { 罾 }
$$




\begin{tabular}{|l|c|c|}
\hline 2.C.2 Cost Basis & $\begin{array}{c}\text { Westinghouse Hanford Company } \\
\text { External Communications } \\
\text { SMSMBS No. 6.10.6 }\end{array}$ & $\begin{array}{c}\text { FY 1995 } \\
\text { Site Support Program Plan } \\
\text { Date Prepared: August 1994 }\end{array}$ \\
\hline
\end{tabular}




\section{EXTERNAL CC}

\section{FORM 2.C.3 FTEs}

6.10 .6

FY 1995 SSPP

\begin{tabular}{|c|c|c|c|c|c|c|c|c|}
\hline \multicolumn{4}{|c|}{ Fu11-Time Equivalent Staff by Job Description } & \multicolumn{5}{|c|}{ NOTE: Job Family only After 1996} \\
\hline JOB FAMILY ? & $\because: \because$ & अ४ & बथ। & : & ४० & ४:। & ४४। & ४ : \\
\hline Job category & 1994 & 1995 & 1996 & 1997 & 1998 & 1999 & 2000 & 2001 \\
\hline MANAGERS $\%$ \% & a & 89 & ४े & $3_{4}$ & 4,8 & अ? & 84. & 44 \\
\hline First line & 5 & 3 & 3 & & & & & \\
\hline General/executive & 2 & 1 & 1 & & & & & \\
\hline \multicolumn{9}{|l|}{ Project/Program } \\
\hline \multicolumn{9}{|l|}{ Other } \\
\hline \multirow{2}{*}{$\begin{array}{l}\text { ENGNNEERS } \\
\text { Chemical }\end{array}$} & ২: & 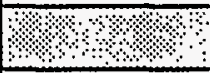 & 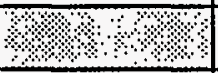 & 1. & 148 & (1) & $1 \%$ & ४ং \\
\hline & & & & & & & & \\
\hline \multicolumn{9}{|l|}{ Civil } \\
\hline \multicolumn{9}{|l|}{ Computer } \\
\hline \multicolumn{9}{|l|}{ Electrical } \\
\hline \multicolumn{9}{|l|}{ Environmental } \\
\hline \multicolumn{9}{|l|}{ Industrial } \\
\hline \multicolumn{9}{|l|}{ Mechanical } \\
\hline \multicolumn{9}{|l|}{ Nuclear } \\
\hline \multicolumn{9}{|l|}{ Petroleum/Mining } \\
\hline \multicolumn{9}{|l|}{ Plant } \\
\hline \multicolumn{9}{|l|}{ Quality Control } \\
\hline \multicolumn{9}{|l|}{ safety } \\
\hline \multicolumn{9}{|l|}{ Other } \\
\hline screntrsts & ४४४४। & : & ४४ঃ। & ४४४।: & ४४ঃ & ঋং & 18..? & ४४ ? \\
\hline Chemists & & & & & & & & \\
\hline
\end{tabular}




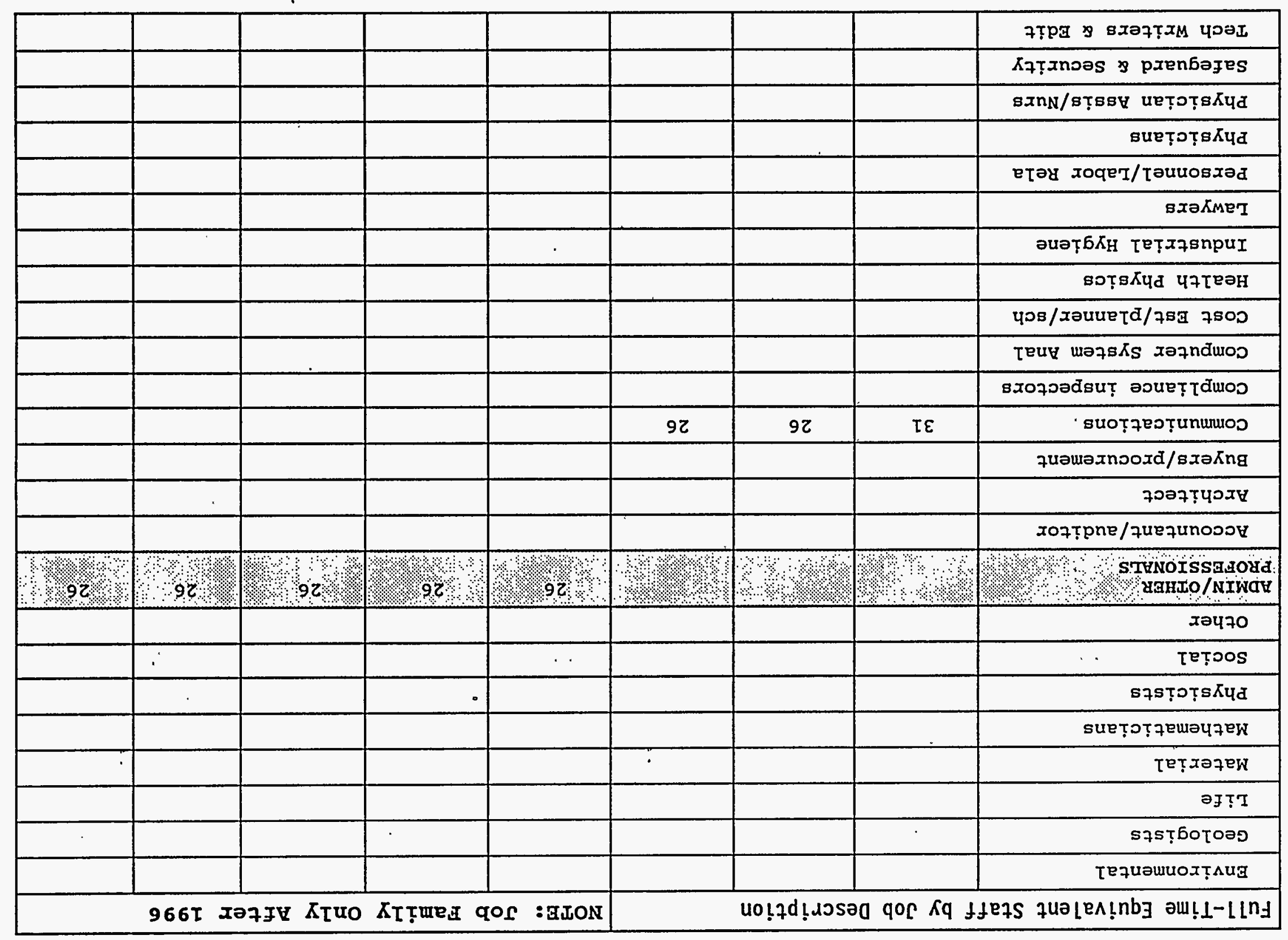




\begin{tabular}{|c|c|c|c|c|c|c|c|c|}
\hline कः & १ै। & १ি॰ & ৪ঃ\%: & ঋ४४। & ४২০। & $4 \%$ & ४४: & 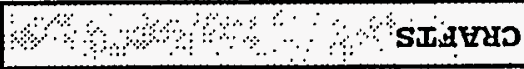 \\
\hline & & & & & & & & хәч70 \\
\hline & $\cdot$ & & & & & & & Yอจ匹 deW/KəAxns \\
\hline & & & & & & & & 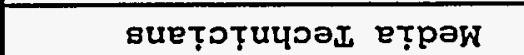 \\
\hline & & & & & & & & 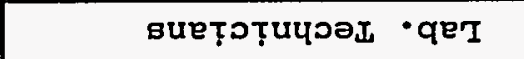 \\
\hline & & & & & & & & 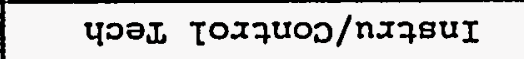 \\
\hline & & & & & & & & 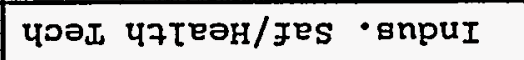 \\
\hline & & & & & & & & 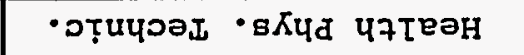 \\
\hline & & & & & & & & 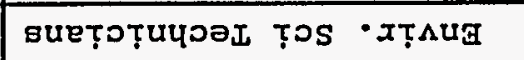 \\
\hline & & & & & & & & 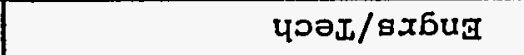 \\
\hline & & & & & & & & sxә7эexd \\
\hline & & & & & & & $\mathrm{I}$ & тәрор/xәđо тә7nđwos \\
\hline భি: & 1\%? & ২২২+: & \%?:ি: & \%ै। & \% & $1 \% . \%:$ & সি। & 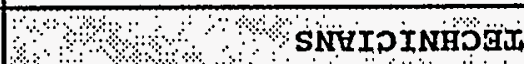 \\
\hline & & & & & & & & тәч70 \\
\hline & & & & & & & & 88อD०xd $\mathrm{pxOM} / 78 T ⿱ \mathrm{~d} d K_{\Psi}$ \\
\hline & & & & . & 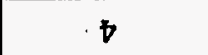 & 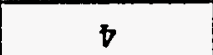 & 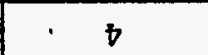 & 8әтฺдвาәxวәа \\
\hline & & & & & & & & 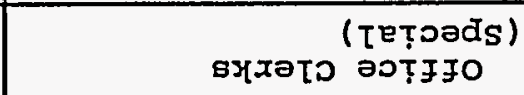 \\
\hline & & & & & $9 \cdot \varepsilon$ & $9 \cdot \varepsilon$ & 9 & 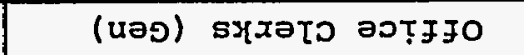 \\
\hline & & & & & & & & sque7sṬssy uṬupy \\
\hline 1948 & \%०० & $19 \%$ & ४. $4 \%$ & $92 \%$ & औ४। & अ४४: & ४४। & 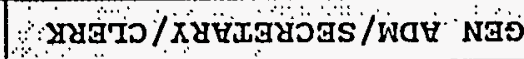 \\
\hline & & & & & & 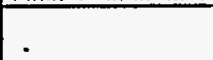 & & Kxобә7ер qог \\
\hline : & + & @ & : & ४ & ४ & के & : & 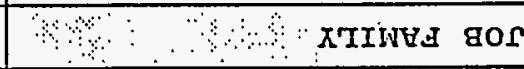 \\
\hline & & & & & & & & Хәч७० \\
\hline & & & & & & & & sxəuțexI \\
\hline \multicolumn{5}{|c|}{ 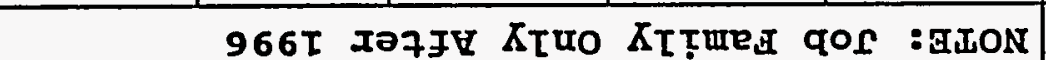 } & \multicolumn{4}{|c|}{ 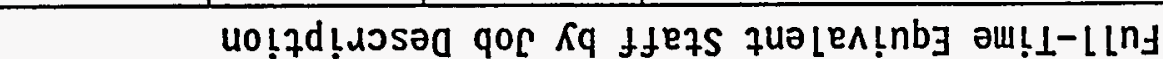 } \\
\hline
\end{tabular}

\section{ddSS G66L 人A}

$9 \cdot 01 \cdot 9$

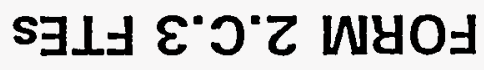

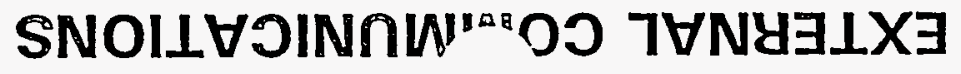




\section{EXTERNAL CG.MUNICATIONS}

FORM 2.C.3 FTEs

6.10 .6

FY 1995 SSPP

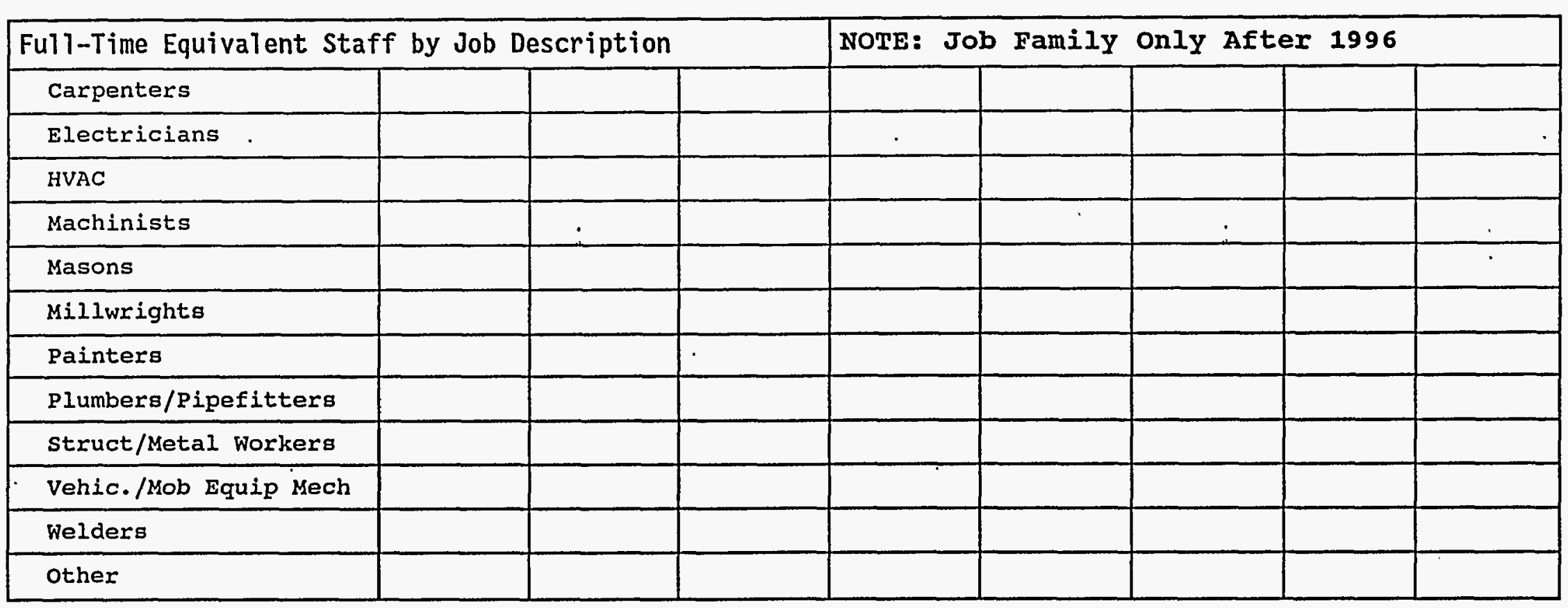




\section{EXTERNAL CG MUNICATIONS}

FORM 2.C.3 FTES

6.10 .6

FY 1995 SSPP

\begin{tabular}{|c|c|c|c|c|c|c|c|c|}
\hline \multicolumn{4}{|c|}{ Ful1-Time Equivalent Staff by Job Description } & \multicolumn{5}{|c|}{ NOTE: Job Family Only After 1996} \\
\hline OPERATORS S & औद & क्ष & थि & ४ & ग४৪ & मे० & क०० & \%४ \\
\hline Chemical system & $\cdot$ & & & & & & & \\
\hline Drillers & & & & & & & & \\
\hline Lt. Vehicle Drivers & . & & . & & . & & & \\
\hline Material Moving Equip & & & & & & & & \\
\hline Nuclear Plant & & & & & & & & \\
\hline Utilities Waste Proces & & & & & & & & \\
\hline other & & & & & & & & \\
\hline LABOR \& GEN WORKERS & ०४! & अ०: & \%४ & ४ & मे & - ४४४: & ४) & $88 \%$ \\
\hline Firefighters & & & & & & & & \\
\hline Food Service & & & & & - & & & \\
\hline Hand/Help Lab Gen & & & & & & & & \\
\hline Hand/Help Lab Spec & & & & & & & & \\
\hline Janitors/Cleaners & & & & & & & & \\
\hline Laundry Workers & & & & & & & & \\
\hline Security Guards & & & & & & & & \\
\hline other & & & & & & & & \\
\hline
\end{tabular}




\begin{tabular}{|c|c|c|c|c|c|}
\hline \multirow{5}{*}{$\begin{array}{l}\text { Work } \\
\text { Breakdown } \\
\text { Structure } \\
\text { Dictionary }\end{array}$} & \multirow{5}{*}{\multicolumn{3}{|c|}{$\begin{array}{c}\text { Westinghouse Hanford Company } \\
\text { EXTERNAL COMMUNICATIONS } \\
\text { Part I - Summary } \\
\text { (Dollars in 000's) }\end{array}$}} & \multirow{7}{*}{$\begin{array}{l}\text { FY' } 1995 \text { SSPP } \\
\text { Rev. \# } 0\end{array}$} & \multirow{2}{*}{\begin{tabular}{|c|} 
Funding ? \\
G\&A $\mathrm{X}$ \\
\end{tabular}} \\
\hline & & & & & \\
\hline & & & & & \begin{tabular}{|l|} 
G\&A X \\
SWS \\
\end{tabular} \\
\hline & & & & & OST \\
\hline & & & & & $\mathrm{DOH}$ \\
\hline Cost Account & \multirow{2}{*}{\multicolumn{3}{|c|}{$\begin{array}{l}\text { Cost Account Title } \\
\text { EXTERNAL COMMUNICATIONS }\end{array}$}} & & POOL \\
\hline $1 \mathrm{MDO} 02$ & & & & & DIRECT \\
\hline $\begin{array}{l}\text { SMS WBS } \\
6.10 .6\end{array}$ & \multicolumn{3}{|c|}{$\begin{array}{l}\text { SMS TIIle } \\
\text { PUBLIC INFORMATION }\end{array}$} & \multirow{2}{*}{\multicolumn{2}{|c|}{$\begin{array}{c}\text { Annualized Rate } \\
\text { (For Organizational Overhead and } \\
\text { Rated Service Pool Use Only) }\end{array}$}} \\
\hline \multicolumn{2}{|l|}{ CAM Review/Approval } & \multicolumn{2}{|r|}{ Date } & & \\
\hline \multicolumn{2}{|l|}{ SMS Program Manager Review/Approval } & \multicolumn{2}{|r|}{ Date } & FY 1994 Rate & FY 1995 Rate Request \\
\hline \multicolumn{2}{|l|}{ Financial Manager Review/Approval } & \multicolumn{2}{|r|}{ Date } & & \multirow[b]{2}{*}{ FY 1995 Approved Rate } \\
\hline Responsible Analyst & & & Date & FY 1995 Target Rate & \\
\hline \multicolumn{2}{|l|}{ OSBRB Review/Approval } & \multicolumn{2}{|r|}{ Date } & & \\
\hline & \multicolumn{2}{|c|}{ FY1994 } & \multicolumn{2}{|c|}{ 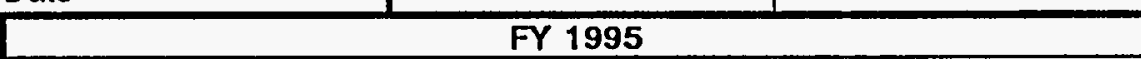 } & \\
\hline FULL-TIME EQUIVALENTS (FTES) & Budget & \begin{tabular}{|c|} 
Fiscal Year Spending \\
Forecast (FYSF)
\end{tabular} & Target & Request & $\begin{array}{c}\text { Approved } \\
\text { Baseline }\end{array}$ \\
\hline . Organizational - Exempt & 38.6 & 36.4 & & 26.7 & \\
\hline . Organizational - Nonexempt & 13.1 & 11.1 & & 8.6 & \\
\hline . Organizational - Bargaining & 0.5 & 0.5 & & 0.4 & \\
\hline Total Organizational FTEs & 52.2 & 48 & & 35.7 & \\
\hline Support FTEs & 0 & 0.7 & & 1.2 & \\
\hline TOTALFTES & 52.2 & 48.7 & & 36.9 & \\
\hline COST ELEMENTS & $\vdots$ & & & $\therefore \quad$ & \\
\hline Labor - Regular & 2,847 & 2,606 & 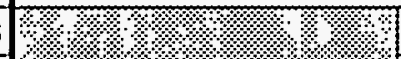 & 2,249 & \\
\hline . Labor - Overtime & 63 & 59 & & 40 & \\
\hline 0 Total Labor & 2,910 & 2,664 & & 2,289 & \\
\hline 1 Materials & 127 & 139 & & 99 & \\
\hline 2 Purchased Services & 859 & 880 & & 891 & ४ \\
\hline 3 Other Hanford & 26 & 33 & 4 & 15 & \\
\hline 4 Site Services & 360 & 371 & & 259 & \\
\hline 5 Internal Charges & 65 & 39 & & 34 & \\
\hline 6 IAM Support & 662 & 845 & & 388 & \\
\hline 7 Overheads & 7 & 38 & & 4 & \\
\hline 8 Revenue & 0 & 0 & $\sqrt{2.3}$ & 0 & \\
\hline TOTALDOLLARS & 5,016 & 5,009 & 3,106 & 3,978 & \\
\hline
\end{tabular}




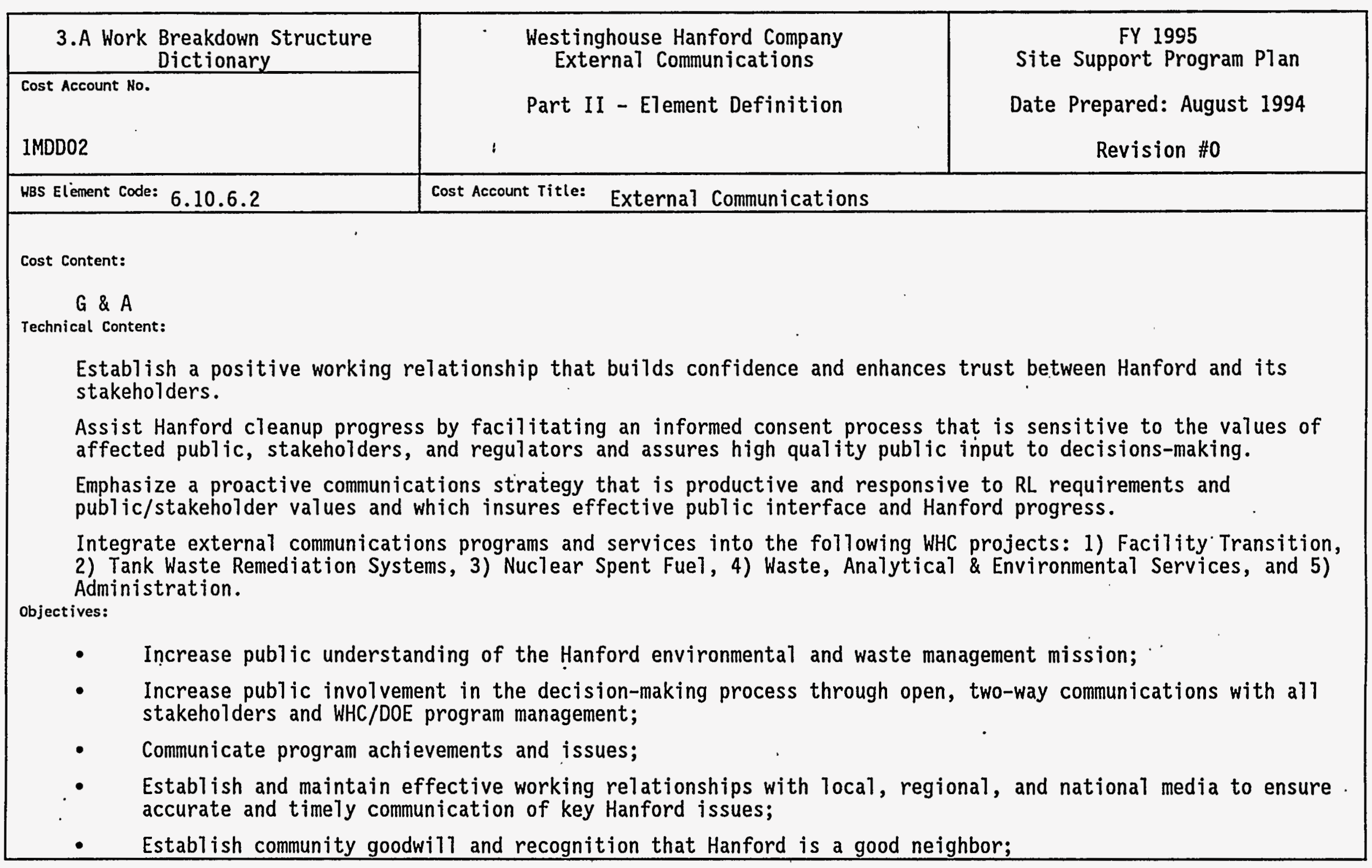


UBS Element code: 6.10 .6 .2

Cost Account Title:

Assumptions/Constraints:

The External Communications Department's goals, objectives and strategies are driven primarily by the Hanford Strategic Plan and the Tri-Party Agreement, both of which cal for Hanford to build partnerships with its stakeholders, and the commitment by Department of Energy and Westinghouse leaders to assure stakeholder participation in Hanford's decision-making process.

The draft Hanford Site Communications Plan also provides a basis with its emphasis on public participation and feedback, enhancing public understanding of Hanford's mission and making public access to information and facilities easier.

The development of the department's goals, objectives and strategies also assumes that staffing will be consistent with funding levels; funding will be received from the ERC for communications services provided to it; and the process through which the three co-regulator tribes will be involved in decision-making will be clarified.

The External Communications Department's achievement of its mission will depend greatly on something beyond its control: the ability of the Department of Energy and Westinghouse to prevent events which damage working relationships and reduce stakeholder confidence in Hanford.

Management decisions intended to be positive also may become an issue at the External Communications level. A key example is the recent decision about tribal interaction and involvement in the decision-making process, which may require more public involvement and other communications support than has been anticipated.

Providing direct communications response to an unanticipated event will likely use resources earmarked for other

planned products and services. There is little choice, however, for an ineffective communications response Milestones:

likely would add to the damage caused by the event or decision to which the department was responding.

$N / A$

Deliverables:

1. Revitalize a pro-active Hanford Speakers' Bureau. Due: $12 / 94$

2. Develop a proposal for two reports; the first reflecting Hanford progress highlights and the second report showing progress tied to the Hanford Strategic Plan success indicators. Due: $10 / 94$

3. Reintroduce proposals for open house and expanded tour program. Due: $12 / 94$ 
\begin{tabular}{|l|l|}
\hline HBS Element Code: & Cost Account Title: External Communications
\end{tabular}

4. Actively support $D O E-H Q$ 's declassification initiative by communicating internal/externally what documents have been declassified.

Due: Ongoing

5. Actively seek out and use new state-of-the-art communication technologies to enhance Hanford's responsiveness to public information requests and improve public access to information. (e.g. Internet, mult imedia CD ROM)

Due: Ongoing

6. Develop a proposal for a program that will educate Hanford employees about Tribal culture and values, and its relationships to the Hanford Site. Due: $2 / 95$

7. Assist DOE-RL to build separate, but equal relationships with Native American tribes so that regular and meaningful involvement occurs on a government-to-government basis. Due: Ongoing

8. Assist DOE-RL management in conducting regular Tribal government meetings. Due: As required

9. Assist DOE-RL in maintaining decision-drive public involvement (e.g., the Hanford Advisory Board) by counseling program management and providing administrative, logistical and communications support. Due: Ongoing

10. Provide input to DOE-RL for the development of a sitewide public involvement policy. Due: First Quarter, FY95

11. Support DOE-RL in the implementation of a sitewide public involvement policy by developing a public involvement strategy and plan. Both will attempt to integrate all DOE decisions and public involvement processes, both inside and outside the Tri-Party Agreement.

- Strategy

Due: Second Quarter, FY95

- Plan

Due: Second Quarter, FY95

12. Facilitate the success of the Hanford Advisory Board to promote meaningful public input on Hanford decisions:

- Facilitate information flow between Board members and Hanford management and programs Due: Ongoing 


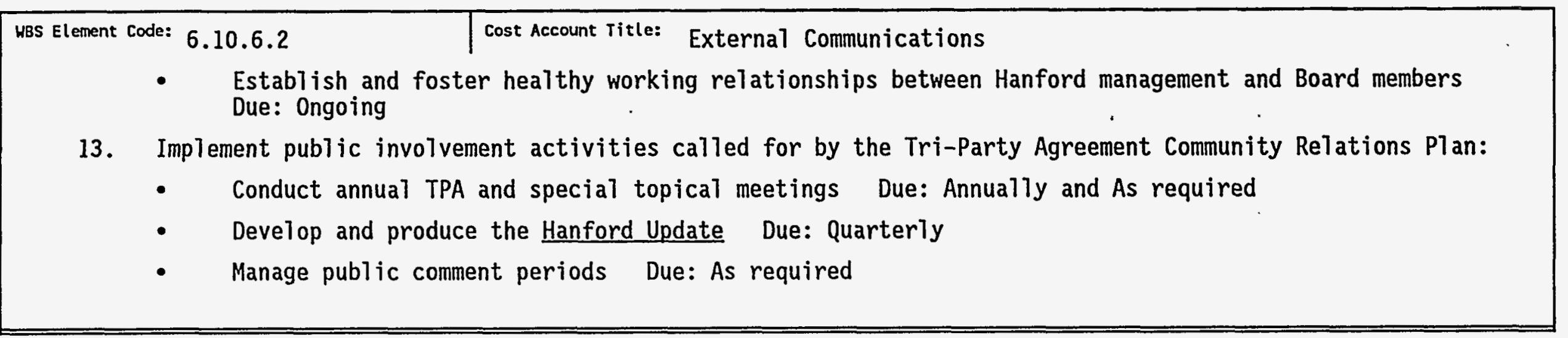




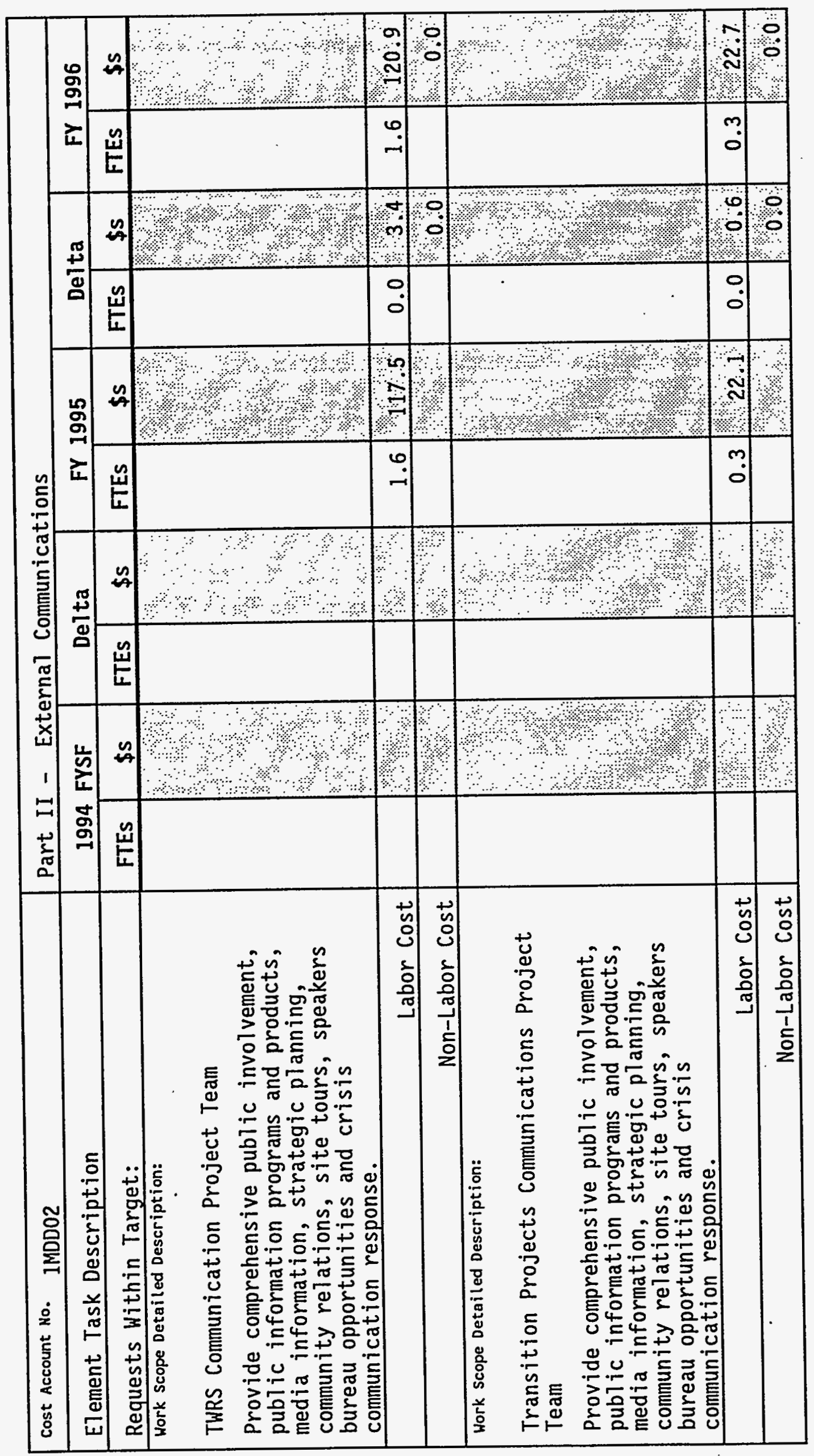




\begin{tabular}{|c|c|c|c|c|c|c|c|c|c|c|}
\hline \multirow{3}{*}{$\begin{array}{l}\text { Cost Account Mo. IMDD02 } \\
\text { Element Task Description } \\
\text { Requests Within Target: }\end{array}$} & \multicolumn{10}{|c|}{ Part II - External Communications } \\
\hline & \multicolumn{2}{|c|}{1994 FYSF } & \multicolumn{2}{|c|}{ Delta } & \multicolumn{2}{|c|}{ FY 1995} & \multicolumn{2}{|c|}{ Delta } & \multicolumn{2}{|c|}{ FY 1996} \\
\hline & FTES & $\$ s$ & FTES & $\$ s$ & FTES & $\$ s$ & FTES & $\$ s$ & FTES & $\$ s$ \\
\hline $\begin{array}{l}\text { Hork Scope Detailed Description: } \\
\text { Spent Nuclear Fuels Project Team } \\
\text { Provide comprehensive public involvement, } \\
\text { public information programs and products, } \\
\text { media information, strategic planning, } \\
\text { community relations, site tours, speakers } \\
\text { bureau opportunities and crisis } \\
\text { communication response. }\end{array}$ & & \& & & & & & & ? & & \\
\hline Labor Cost & & $1 \%$ & & @। & 0.7 & \%55.1: & 0.0 & 1.6 & 0.7 & 56.7 \\
\hline Non-Labor Cost & & : & & @: & & : & & 0.0 & & 80.0 \\
\hline $\begin{array}{l}\text { Hork Scope Detailed Description: } \\
\text { Waste, Analysis and Environmental } \\
\text { Communications Project Team } \\
\text { Provide comprehensive public involvement, } \\
\text { public information programs and products, } \\
\text { media information, strategic planning, } \\
\text { community relations, site tours, speakers } \\
\text { bureau opportunities and crisis } \\
\text { communication response. }\end{array}$ & & $\begin{array}{ll}4 \\
8 \\
8\end{array}$ & & (1) & & 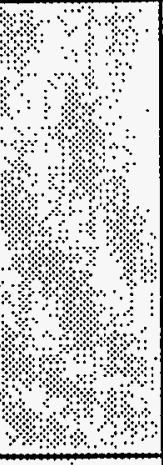 & & 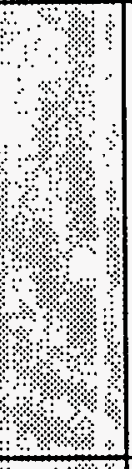 & & 4 \\
\hline Labor Cost & & 8 & & \% & 0.2 & 14,7 & 0.0 & 0.4 & 0.2 & $15: 1$ \\
\hline Non-Labor Cost & & $28 \%$ & & अे & & ४४। & & 0.0 & & 0.0 \\
\hline
\end{tabular}




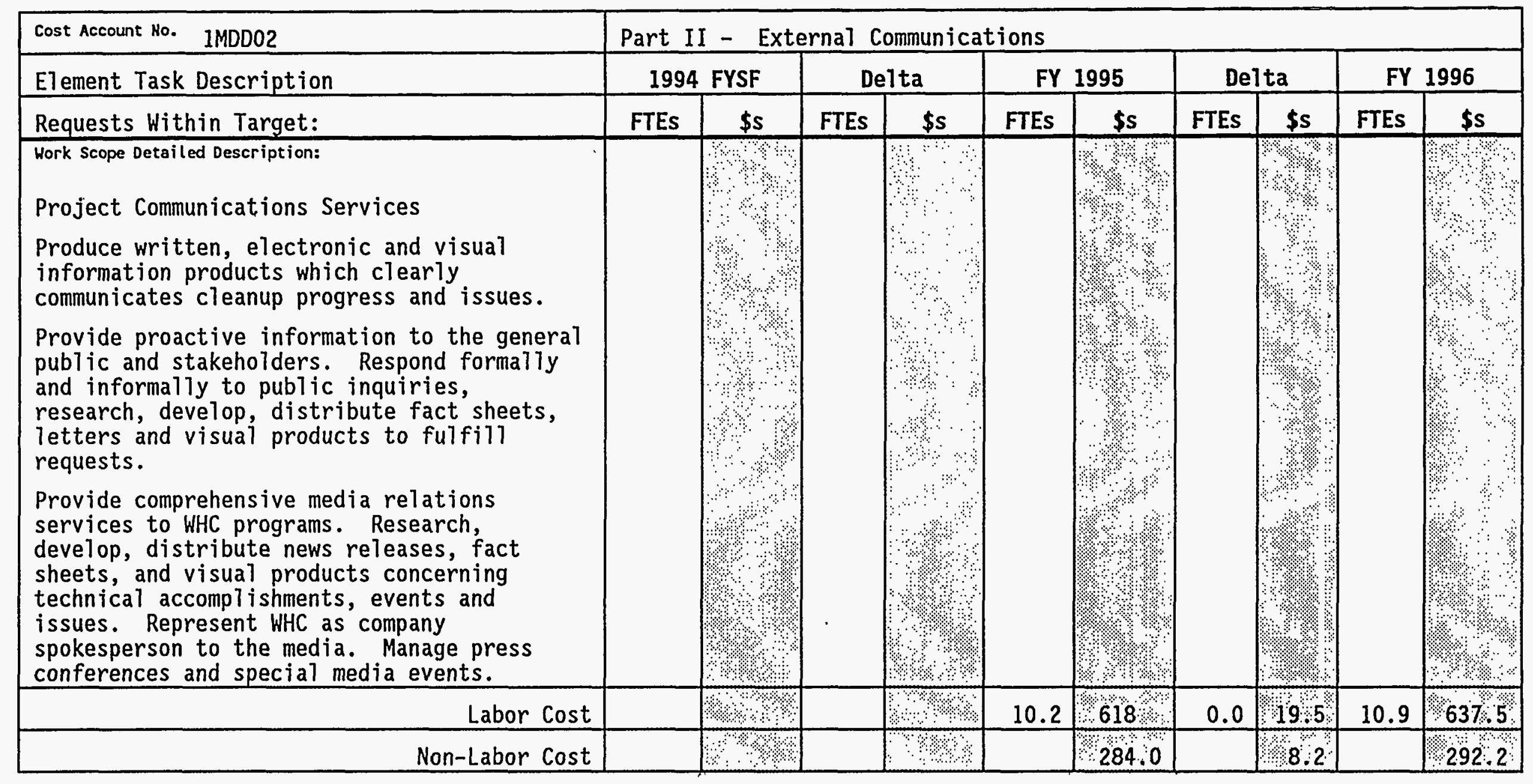




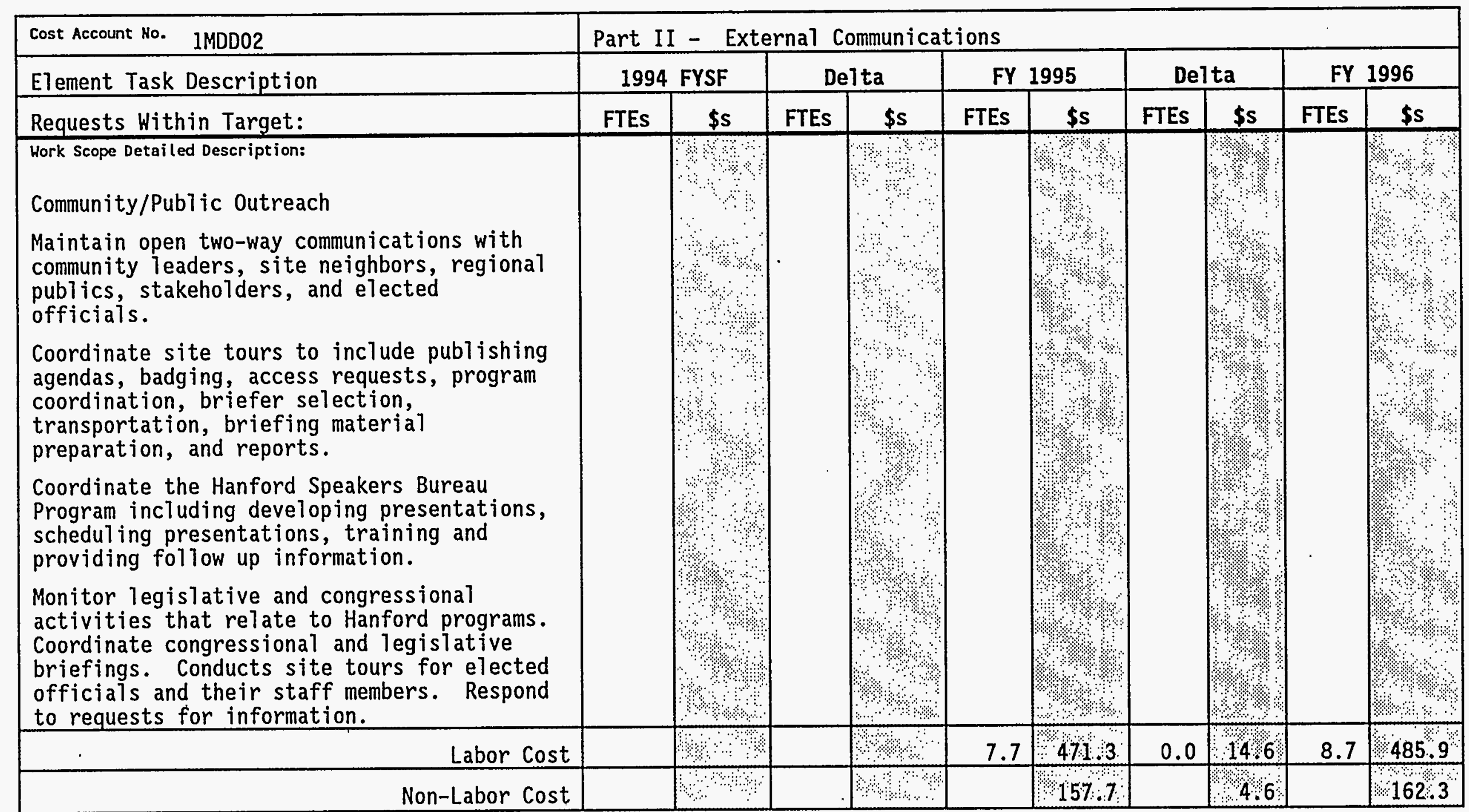




\begin{tabular}{|c|c|c|c|c|c|c|c|c|c|c|}
\hline \multirow{3}{*}{$\begin{array}{l}\text { Cost Account No. } 1 \mathrm{MDDO2} \\
\text { Element Task Description } \\
\text { Requests Within Target: }\end{array}$} & \multicolumn{10}{|c|}{ Part II - External Communications } \\
\hline & \multicolumn{2}{|c|}{1994 FYSF } & \multicolumn{2}{|c|}{ Delta } & \multicolumn{2}{|c|}{ FY 1995} & \multicolumn{2}{|c|}{ Delta } & \multicolumn{2}{|c|}{ FY 1996} \\
\hline & FTES & $\$ s$ & FTES & $\$ s$ & FTEs & $\$ s$ & FTES & $\$ \mathbf{s}$ & FTEs & $\$ s$ \\
\hline Work Scope Detailed Description: & & & & & & & & & & \\
\hline Public Involvement Services & & & & & & & & & & \\
\hline $\begin{array}{l}\text { Plan, coordinate, and conduct public } \\
\text { involvement activities which 1) support } \\
\text { program decisions and } 2 \text { ) assure compliance } \\
\text { with the spirit and intent of environmental } \\
\text { laws and agreements with regulators and } \\
\text { stakeholders. }\end{array}$ & & & & & & 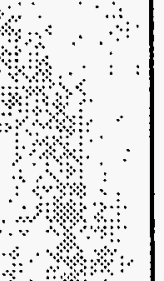 & & ?: & & : \\
\hline $\begin{array}{l}\text { Facilitate the strategic anatysis process } \\
\text { that identifies key decisions that need } \\
\text { stakeholder participation. }\end{array}$ & & & & & & & & $\begin{array}{lll} & 1 \\
\end{array}$ & & 8 \\
\hline $\begin{array}{l}\text { Serve as WHC liaison with stakeholders and } \\
\text { special interest groups. Arrange } \\
\text { appropriate interaction forums. Initiate } \\
\text { the sharing of project status information } \\
\text { with stakeholders and special interest } \\
\text { groups. }\end{array}$ & & & & & & $\begin{array}{lll}2 \\
4 \\
4\end{array}$ & & ?ै: & & $\begin{array}{ll}4 \\
4\end{array}$ \\
\hline $\begin{array}{l}\text { Provide public involvement and } \\
\text { administrative support to DOE-RL in support } \\
\text { of the Hanford Advisory Board and its four } \\
\text { subcommittees. Activities include preparing } \\
\text { draft issue papers, logistics, meeting notes } \\
\text { and records, cost accounting, travel } \\
\text { services, and meeting management. }\end{array}$ & & $\begin{array}{l}3 \\
1 \% \\
1 \%\end{array}$ & & मे? & & 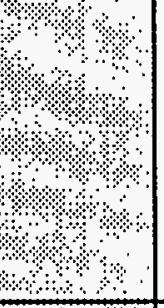 & & (2) & & 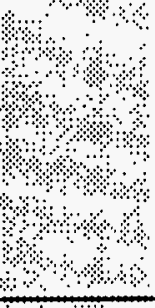 \\
\hline Labor Cost & & ?े। & & १े & 4.8 & 224.9 & 0.0 & 6.5 & 4.8 & 231.4 \\
\hline Non-Labor Cost & & & & & & 174.1 & & \% 5.1 & & 179.2 \\
\hline
\end{tabular}




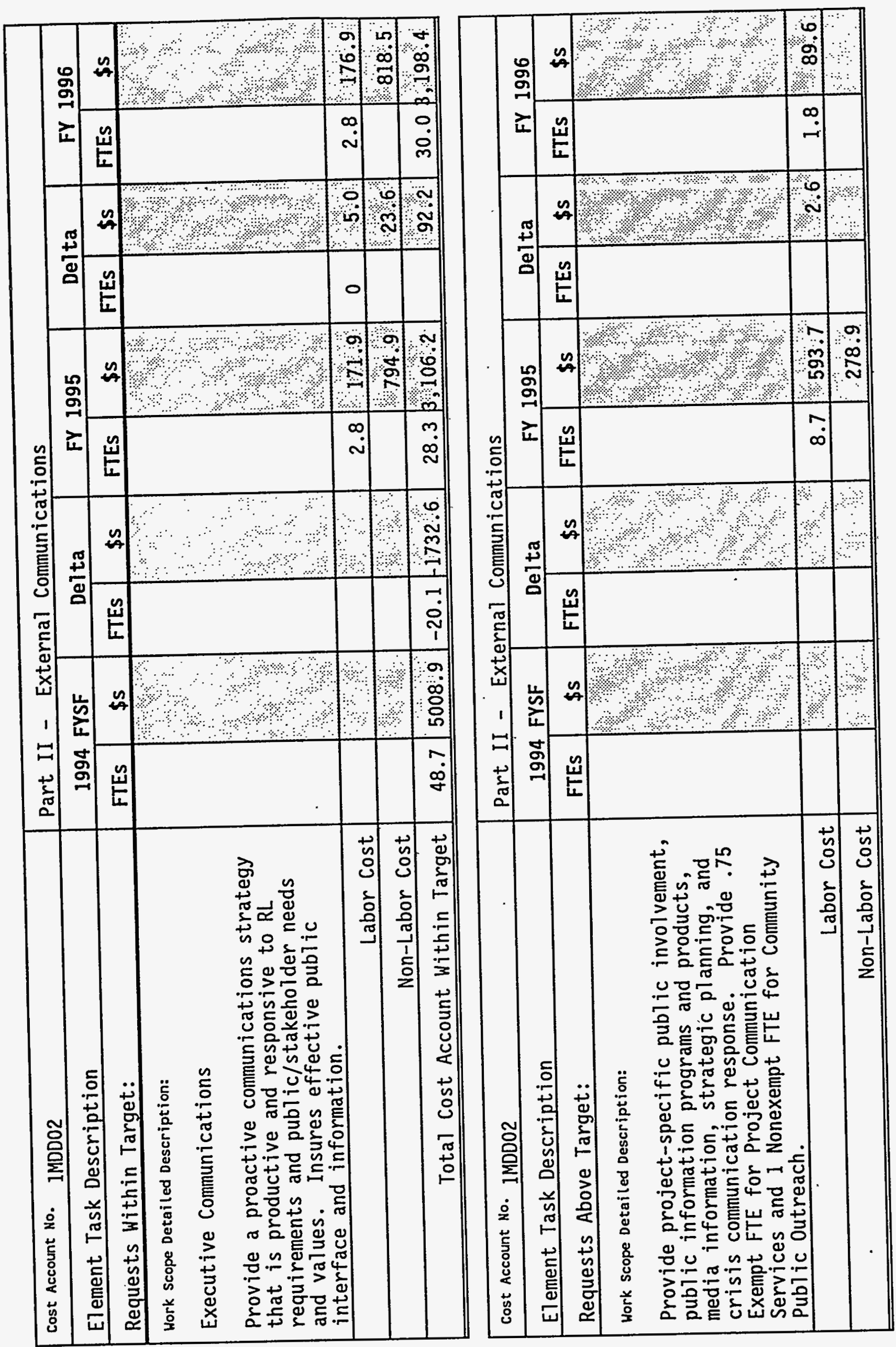




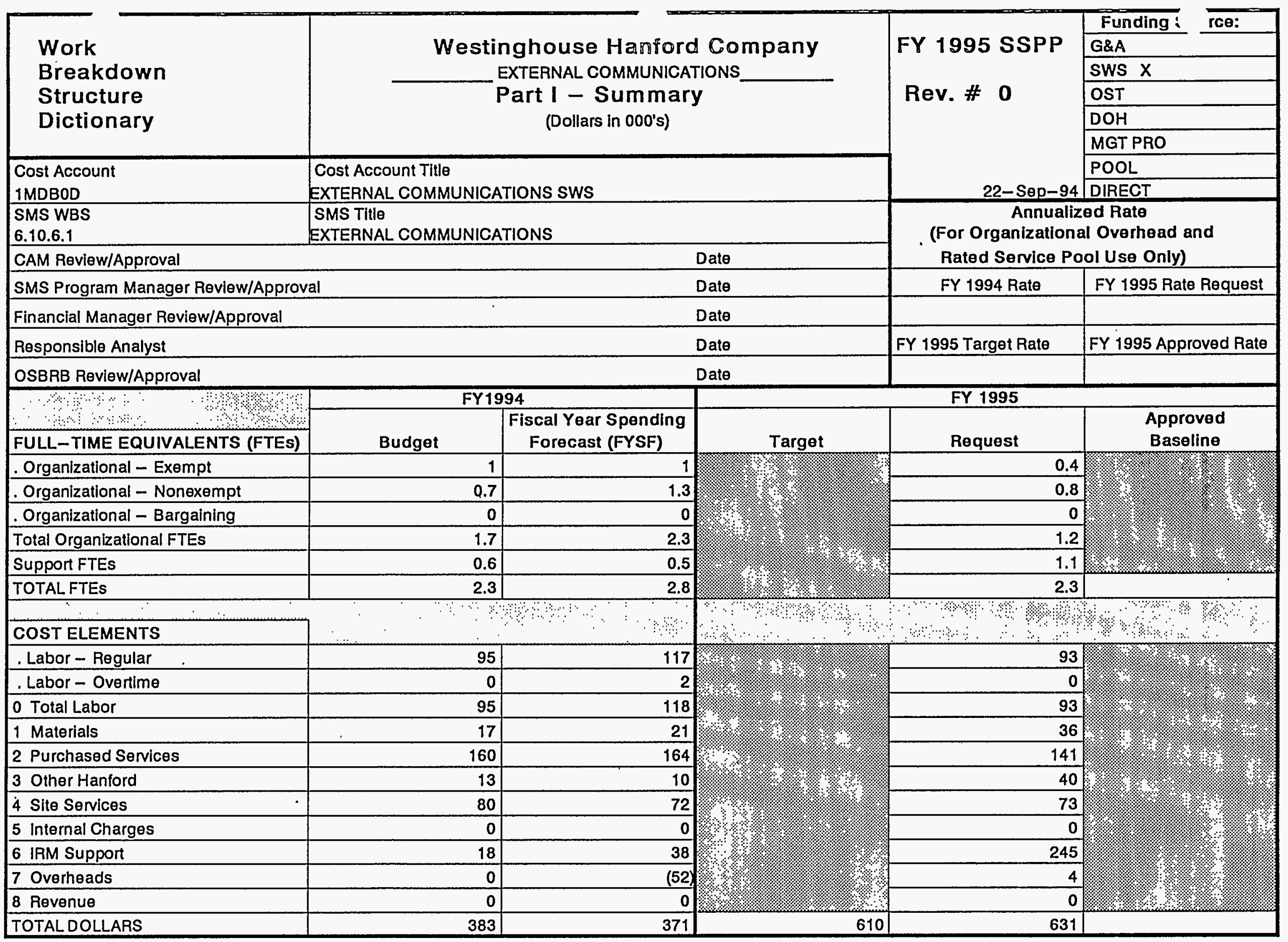




\begin{tabular}{|c|c|c|c|}
\hline \multicolumn{2}{|c|}{$\begin{array}{c}\text { 3.A Work Breakdown Structure } \\
\text { Dictionary }\end{array}$} & \multirow{2}{*}{$\begin{array}{l}\text { Westinghouse Hanford Company } \\
\text { External Communications } \\
\text { Part II - Element Definition }\end{array}$} & \multirow{2}{*}{$\begin{array}{l}\text { FY } 1995 \\
\text { Site Support Program Plan } \\
\text { Date Prepared: August } 1994 \\
\text { Revision \#0 }\end{array}$} \\
\hline Cost Account No & & & \\
\hline \multicolumn{2}{|c|}{ HBS Element Code: 6.10 .6 .1} & \multicolumn{2}{|l|}{ Cost Account ritle: External Communications } \\
\hline \multicolumn{4}{|c|}{$\begin{array}{l}\text { External Communications SWS cost account is funded by SWS. } \\
\text { Technical content: }\end{array}$} \\
\hline \multicolumn{4}{|c|}{$\begin{array}{l}\text { Provide site wide communications support services, such as the Hanford daily newsclips, to DOE-RL, DOE-HQ, and } \\
\text { contractor Senior Management. Management of the Hanford Emergency Public Information Program to include } \\
\text { operation of the Joint Information Center in compliance with DOE order 5500.4B. Provides site wide multi-media } \\
\text { products and services. }\end{array}$} \\
\hline \multirow{2}{*}{\multicolumn{4}{|c|}{$\begin{array}{l}\text { - Provide a high-quality news clip package to DOE-RL and contractor manager each day. } \\
\text { - Improve the effectiveness of Hanford's emergency public information capabilities. } \\
\text { - Provide equipment, training, and facilitator at the Joint Information Center (JIC). } \\
\text { - Provide quality multi-media products which commemorate a total, complete Hanford clean-up story. } \\
\text { - Production of the multi-media products (CD-ROM) is new scope directed by DOE-HQ and approved by DOE-RL } \\
\text { communications. }\end{array}$}} \\
\hline & & & \\
\hline \multicolumn{4}{|c|}{$\begin{array}{l}\text { Milestones: } \\
\text { N/A } \\
\text { Deliverables: }\end{array}$} \\
\hline \multicolumn{4}{|c|}{ Joint Information Center } \\
\hline 1. & & 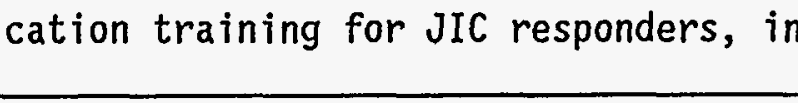 & \\
\hline
\end{tabular}




\begin{tabular}{|c|c|}
\hline \multicolumn{2}{|r|}{ Cost Account Title: External Communications } \\
\hline 2. & $\begin{array}{l}\text { Provide comprehensive Emergency Public Information training to all new JIC responders. } \\
\text { Due: Annually }\end{array}$ \\
\hline 3. & $\begin{array}{l}\text { Maintain a fuTl state of response readiness for the JIC to include facilities, procedures, and personnel. } \\
\text { Due: Ongoing }\end{array}$ \\
\hline 4. & $\begin{array}{l}\text { Activate and participated full in all field exercised conducted by DOE-RL. } \\
\text { Due: Quarterly }\end{array}$ \\
\hline 5. & $\begin{array}{l}\text { Maintain RLEP-12, Public Affairs Emergency Plan and Procedures, in compliance with D0E Order } 5500.4 B \text { and } \\
\text { other guidance. } \\
\text { Due: Ongoing }\end{array}$ \\
\hline 6. & $\begin{array}{l}\text { Manage the JIC corrective action program. } \\
\text { Due: Ongoing }\end{array}$ \\
\hline 7. & $\begin{array}{l}\text { Provide staff and support to RL and HQ EPI programs and initiatives. } \\
\text { Due: Ongoing }\end{array}$ \\
\hline 8. & $\begin{array}{l}\text { Support the Washington Public Power Supply System as requested. } \\
\text { Due: Quarterly }\end{array}$ \\
\hline \multicolumn{2}{|c|}{ Newscl ips } \\
\hline 1. & $\begin{array}{l}\text { Provide daily news clips to RL and contractor management and program offices. } \\
\text { Due: Ongoing }\end{array}$ \\
\hline 2. & $\begin{array}{l}\text { Maintain a media file of clippings. } \\
\text { Due: Ongoing }\end{array}$ \\
\hline \multicolumn{2}{|c|}{ Visual Products } \\
\hline 1. & $\begin{array}{l}\text { Develop and distribute multi-media products (CD-ROM) that identify the challenges and successes involved } \\
\text { in Hanford clean up. (new scope) } \\
\text { Due: Ongoing }\end{array}$ \\
\hline 2. & $\begin{array}{l}\text { Produce and support a new.Hanford exhibit. (new scope) } \\
\text { Due: Ongoing }\end{array}$ \\
\hline
\end{tabular}


HBS Element Code: 6.10 .6 .1

Cost Account Title: External Communications

3. Produce a new Hanford video. (new scope)

Due: Annualiy

4. Produce and insert into the Tri-City Herald two supplements. (new scope) Due: Semi-annuat7y 


\begin{tabular}{|c|c|c|c|c|c|c|c|c|c|c|}
\hline \multirow{3}{*}{$\begin{array}{l}\text { Cost Account No. } \\
\text { Element Task Description } \\
\text { Requests Within Target: }\end{array}$} & \multicolumn{10}{|c|}{ Part II - } \\
\hline & \multicolumn{2}{|c|}{1994 FYSF } & \multicolumn{2}{|c|}{ Delta } & \multicolumn{2}{|c|}{ FY 1995} & \multicolumn{2}{|c|}{ Delta } & \multicolumn{2}{|c|}{ FY 1996} \\
\hline & FTES & $\$ \mathbf{s}$ & FTES & $\$ \mathbf{s}$ & FTES & $\$ \mathbf{s}$ & FTES & \$s & FTEs & \$s \\
\hline Hork Scope Detailed Description: & & & & & & & & & & \\
\hline Emergency Public Information & & & & & & & & & & \\
\hline $\begin{array}{l}\text { Manage the Hanford Joint Information Center } \\
\text { (JIC) in compliance with DOE Order } 5500.4 \mathrm{~B} \\
\text { using a } .3 \text { exempt FTE, a WHC retiree }(1000 \\
\text { hrs), and .5 FTE clerical support. }\end{array}$ & & & & & & & & धै? & & \\
\hline $\begin{array}{l}\text { Maintain a state of readiness in personnel, } \\
\text { training, procedures, and facilities which } \\
\text { will assure prompt, accurate and timely } \\
\text { release of vital information from DOE-RL, } \\
\text { site contractors and affected counties and } \\
\text { states from the JIC. Non-labor costs } \\
\text { include training consultants (60k), PNL } \\
\text { technical editors (30k), materials (36k), } \\
\text { and map making services (25k). Occupancy, } \\
63.5 \text {. }\end{array}$ & & & & 's & & 84 & & ?: & & e \\
\hline Labor Cost & 2.0 & 88.1 & -.6 & -26.2 & 1.4 & 62.6 & -0.0 & 1.8 & 1.4 & 64.4 \\
\hline Non-Labor Cost & & 229.5 & & -116 & & 217.9 & & 6.3 & & 224.2 \\
\hline $\begin{array}{l}\text { Hork Scope Detailed Description: } \\
\text { Daily News Clips } \\
\text { Provide daily news clips with one non-exempt } \\
\text { working } 32 \text { hours per week. This function } \\
\text { requires subscriptions to approximately } 35 \\
\text { periodicals (10k). }\end{array}$ & & ? & & है। & & 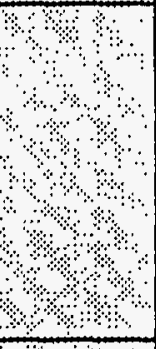 & & 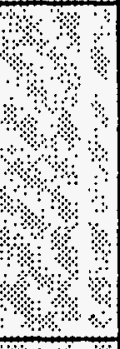 & & \\
\hline Labor Cost & 0.9 & 29.6 & -.1 & -4.6 & 0.8 & 25.0 & 0.0 & 0.7 & 0.8 & 25.7 \\
\hline Non-Labor cost & & 23,0 & & $-13: 5$ & & 9.5 & & 0.3 & & \\
\hline
\end{tabular}




\begin{tabular}{|c|c|c|c|c|c|c|c|c|c|c|}
\hline \multirow{3}{*}{$\begin{array}{l}\text { Cost Account Ho. } \\
\text { Element Task Description } \\
\text { Requests Within Target: }\end{array}$} & \multicolumn{10}{|c|}{ Part II - } \\
\hline & \multicolumn{2}{|c|}{1994 FYSF } & \multicolumn{2}{|c|}{ Defta } & \multicolumn{2}{|c|}{ FY 1995} & \multicolumn{2}{|c|}{ Delta } & \multicolumn{2}{|c|}{ FY 1996} \\
\hline & FTES & $\$ s$ & FTEs & \$s & FTEs & $\$ s$ & FTEs & $\$ s$ & FTEs & $\$ s$ \\
\hline $\begin{array}{l}\text { Hork Scope Detailed Description: } \\
\text { NEW SCOPE } \\
\text { Project Communications Information Products } \\
\text { Develop and distribute multi-media } \\
\text { information products (CD-ROM) (196k), create } \\
\text { a new Hanford site wide exhibit (25k), } \\
\text { produce a site wide Hanford video (30k), and } \\
\text { travel and expenses to support the above } \\
\text { (44k). }\end{array}$ & & 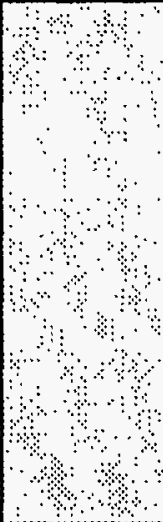 & & 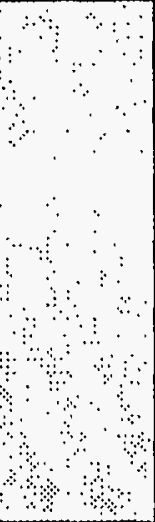 & & 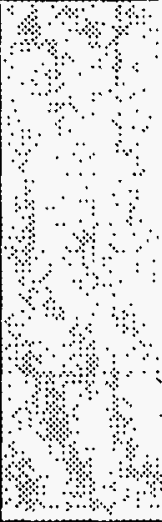 & & $\begin{array}{l}3 \\
3 \\
4 \\
4\end{array}$ & & 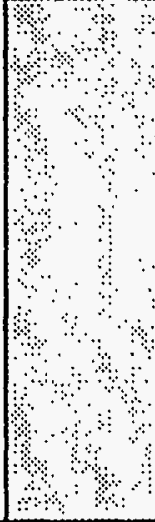 \\
\hline Labor Cost & & ध & & $\%$ & & ?: & & अ.:० & & : \\
\hline Non-Labor Cost & & एँ' & & \& & & 29500 & & 8,6 & & 303.6 \\
\hline Requests Above Target & & क् & & $8:$ & & ॥। & & : $:$ & & $8 \%$ \\
\hline $\begin{array}{l}\text { Hork Scope Detailed Description: } \\
\text { NEW SCOPE } \\
\text { DeveTop and distribute a four page semi- } \\
\text { annuat progress report. }\end{array}$ & & 3. & & $\begin{array}{l}0 \\
4 \\
3 \\
3\end{array}$ & & 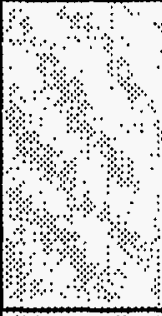 & & 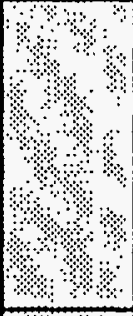 & & : \\
\hline Labor Cost & & ०ै। & 0.1 & 85.0 & 0.1 & 2450 & 0.0 & 0,1 & 0.1 & 5.1 \\
\hline Non-Labor Cost & & भै। & " & 160 & & 16,0 & & मे: & 0.5 & 16.5 \\
\hline Total Cost Account & 2.9 & 370.9 & -0.6 & 260.1 & 2.3 & $631: 0$ & -0.0 & 18.3 & 2.3 & 649.3 \\
\hline
\end{tabular}



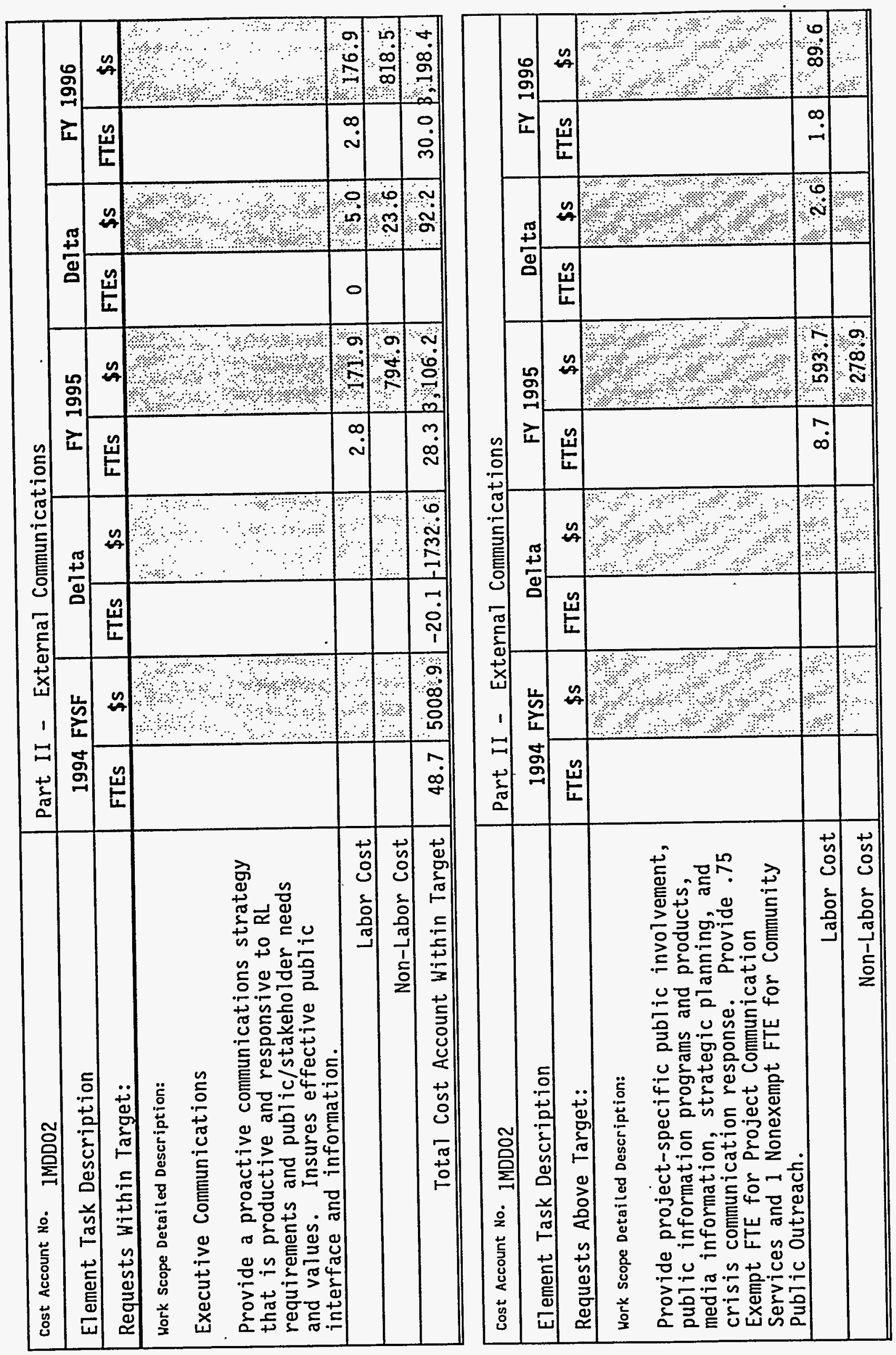